\title{
LncRNA RHPN1-AS1 Inhibition Induces Autophagy and Apoptosis in Prostate Cancer Cells via the miR- 7-5p/EGFR/PI3K/AKT/mTOR Signaling Pathway
}

\section{Xiu-Long Ma}

The Second Affiliated Hospital of Xi'an Jiaotong University

Hong-Tao Ren

The Second Affiliated Hospital of Xi'an Jiaotong University

\section{Yang Zhang}

The Second Affiliated Hospital of Xi'an Jiaotong University

\section{Bao-Feng Wang}

The Second Affiliated Hospital of Xi'an Jiaotong University

\section{Hong-Bing Ma ( $\nabla$ hongbm@aliyun.com )}

The Second Affiliated Hospital of Xi'an Jiaotong University https://orcid.org/0000-0001-9063-4119

\section{Research Article}

Keywords: Prostate cancer, LncRNA RHPN1-AS1, autophagy, apoptosis, the miR-7-

5p/EGFR/PI3K/AKT/mTOR signaling pathway

Posted Date: August 2nd, 2021

DOI: https://doi.org/10.21203/rs.3.rs-723604/v1

License: (c) (1) This work is licensed under a Creative Commons Attribution 4.0 International License. Read Full License 


\section{Abstract}

IncRNA RHPN1-AS1 (RHPN1-AS1) has been confirmed to promote tumor progression in multiple cancers and is upregulated in prostate cancer ( $\mathrm{PCa}$ ), but whether it has an effect on PCa progression remains unclear. In this study, we found that PCa patients with high RHPN1-AS1 expression had a shorter survival time, and RHPN1-AS1 was significantly upregulated in PCa tissues and cells. Based on informatics analysis we predicted that miR-7-5p binds to 3'UTR of RHPN1-AS1 and epidermal growth factor receptor (EGFR) and verified it by luciferase reporter gene assay. Subsequently, we transfected PCa cells with RHPN1-AS1 overexpression vector (RHPN1-AS1), knockdown plasmids (sh-RHPN1-AS1) and/or miR-7-5p mimics or inhibitor and/or overexpression vector (EGFR) or small interfering RNA of EGFR (si-EGFR) or its control, and found that overexpression of RHPN1-AS1 inhibited miR-7-5p expression and promoted EGFR expression, silencing RHPN1-AS1 inhibited proliferation and invasion and induced G2/M arrest, apoptosis and autophagy in PCa cells. 3MA (an inhibitor of autophagy)-mediated autophagy inhibition attenuated RHPN1-AS1 inhibition-induced apoptosis. Overexpression miR-7-5p or silencing EGFR promoted LC3-I to LC3-II conversion, enhanced autophagy activity, induced cleaved-caspase-3 expression and apoptosis in PCa cells. Furthermore, we found that overexpression of RHPN1-AS1 promoted phosphorylation of phosphatidylinositol 3-kinase (PI3K), AKT and mTOR, inhibited LC3-I to LC3-II conversion and reduced apoptosis in PCa cells, while GSK2126458 (an inhibitor of PI3K) reversed the effect of RHPN1-AS1 on PCa cells. In summary, RHPN1-AS1 acted as a ceRNA of miR-7-5p to upregulate EGFR expression, silencing RHPN1-AS1 suppressed PCa tumor progression by inducing autophagy and apoptosis in PCa cells through the miR-7-5p/EGFR/PI3K/AKT/mTOR pathway.

\section{Introduction}

Prostate cancer $(\mathrm{PCa})$ is considered the most common urological malignancy in males and is the leading cause of cancer-related death in the world. Prostate cancer ranks 7th for incidence and 10th for mortality among male malignancies in China[1, 2]. However, as China begins to enter an aging society, the impact of PCa on the lifestyle and life quality of Chinese men has become increasingly prominent. Moreover, the incidence of PCa has been on the rise in recent years due to changes in diet and lifestyle[3,4]. Approximately $10 \%$ of newly diagnosed patients present with locally advanced PCa, and $5 \%$ showed distant metastasis because of the insidious symptoms [5]. Although improvements in PCa diagnostic strategies and multiple therapeutic approaches have led to a significant reduction in PCa related deaths over the past three decades, the prognosis of patients is still not optimistic. Therefore, early diagnosis and intervention of PCa will help to reduce its harm to patients and improve the therapeutic effect. However, even with the in-depth study of PCa, the pathogenesis and causes of PCa have not been fully understood[6]. This situation has led a large number of scholars to continuously search for molecular indicators for early diagnosis of PCa, molecular targets for PCa treatment and molecular markers for the prognosis of PCa.

In recent years, long non-coding RNAs (IncRNAs), mainly transcribed from the genomic intergenic regions have become a research focus in cancers transcriptome. Functionally, they have been demonstrated to be 
participants in various biological processes through various mechanisms from transcription level to posttranscription level[7]. The oncogenic or tumor suppressive roles of IncRNAs have been well characterized in tumorigenesis[8]. To date, several IncRNAs have been defined as PCa specific. Nevertheless, investigation of the function and mechanism of novel IncRNAs is necessary to find functional biomarkers in PCa progression. RHPN1-AS1 is a IncRNA that was differentially expressed in PCa patients and the potential function of RHPN1-AS1 in multiple tumor progression has been reported[9]. For example, RHPN1-AS1 increased the expression of leucine zipper/EF hand transmembrane-containing-1 (LETM1) in ovarian cancer via inhibiting miR-596 expression to activate the FAK/PI3K/AKT signaling pathway to promote tumorigenesis and metastasis[10]. Other studies also demonstrated the oncogenic role of RHPN1-AS1 in cholangiocarcinoma[11], cervical cancer[12], colorectal cancer[13], gastric cancer[14], and hepatocellular carcinoma[15]. Nevertheless, the molecular mechanism of RHPN1-AS1 regulation in PCa tumor progression remains unclear.

Autophagy, a cellular self-eating process, is a catabolic process that delivers cellular proteins and organelles to lysosomes for subsequent degradation[16]. Autophagy plays a key role in the metabolic regulation of cells. Although the process of autophagy improves cell survival under harsh conditions, in many cases autophagy can induce autophagic programmed cell death, also known as type II cell death $[17,18]$. Autophagy and apoptosis pathways are interconnected, and loss of homeostatic balance between these two pathways may lead to cell death[18]. Previous reports have shown that the phosphoinositide 3-kinase (PI3K)/protein kinase B (AKT)/mammalian target of rapamycin (mTOR) pathway, as a key regulator of autophagy, is involved in the initiation and promotion of a range of pathological diseases, including various tumors[19]. In parallel, reports have shown that the PI3K/AKTmTOR signaling pathway is upregulated by loss of phosphatase in $30-50 \%$ of patients with PCa has been reported[20]. Targeting PI3K/AKT-mTOR mediated autophagy is an important therapeutic strategy for PCa and is important for inhibiting tumor cell growth and metastasis in $\mathrm{PCa}[20,21]$. Therefore, it is necessary to gain insight into the molecular mechanisms of autophagy and apoptosis in PCa, search for novel biomarkers for detection, and improve current therapeutic strategies. In this study, we explored the effect of RHPN1-AS1 on the proliferation, apoptosis and autophagy of PCa cells and its mechanism, expecting to provide a new theoretical basis for the treatment of $\mathrm{PCa}$.

\section{Materials And Methods}

\section{Tissue samples}

Twenty-one pairs of PCa tissue samples and adjacent normal tissues were collected from the Second Affiliated Hospital of Xi'an Jiaotong University between June 2019 and December 2020. This study was approved by the Ethics Committees of the Second Affiliated Hospital of Xi'an Jiaotong University, and was carried out in accordance with the principles of the Declaration of Helsinki. Each participant did not receive radiotherapy and chemotherapy before tissue collection and signed written informed consent before the study. All samples were stored at $-80^{\circ} \mathrm{C}$ until required for further analysis. 


\section{Cell culture}

Human PCa cell lines PC-3, LNCaP, DU145 and normal prostate epithelial cell RWPE-1 were purchased from American Type Culture Collection (ATCC; Manassas, VA, USA). All cells were cultured as recommended in DMEM (Gibco, Rockville, MD, USA) containing 1\% antibiotics (Gibco) and 10\% FBS (Gibco) under $5 \% \mathrm{CO}_{2}$ at $37^{\circ} \mathrm{C}$. Before using in this study, all cell lines were authenticated by STR profiling and tested for mycoplasma contamination in June 2019.

\section{Cell transfection}

The RHPN1-AS1 shRNA (sh-RHPN1-AS1) or negative control shRNA (sh-NC), RHPN1-AS1 overexpression vector (RHPN1-AS1) or pcDNA3.1 empty vector (pcDNA3.1), miR-7-5p mimic or NC mimic, miR-7-5p inhibitor or NC inhibitor, small interfering RNA of Epidermal growth factor receptor (EGFR, si-EGFR) or siNC were chemically synthesized and provided by Gene Pharma (Shanghai, China). The plasmid, sh-RNA and siRNAs were transfected into LNCaP and PC3 cells for $48 \mathrm{~h}$ with Lipofectamine 3000 according to the manufacturer's instructions, and RT-qPCR checked the transfection efficiency.

\section{RNA isolation of nuclear and cytoplasmic fractions}

The RHPN1-AS1 content in the cell cytoplasm and cell nucleus of PC3 cells was assayed following the protocol of PARIS ${ }^{\text {TM }}$ Kit (AM1921, Invitrogen). After cells were treated with cell fractionation buffer and then centrifuged, RT-qPCR was applied to determine the expression levels of NNT-AS1 in cytoplasmic and nuclear fractions, with GAPDH (cytoplasmic control) and U6 (nuclear control) as respective controls.

\section{Bioinformatics analysis and dual-luciferase reporter gene assay}

The online tool starBase v2.0 (http://starbase.sysu.edu.cn/) was used to predict the potential target genes and analyze the specific binding sites.

The relationship between miR-7-5p and RHPN1-AS1 or EGFR was verified by dual-reporter gene assay. In brief, the pmirGLO vectors (Promega, Madison, WI, USA) containing the sequences of RHPN1-AS1 wild type with the miR-7-5p binding sites or RHPN1-AS1 mutant with the mutated miR-7-5p binding sites, or containing the sequences of wild type EGFR $3^{\prime}$ untranslated region (UTR) with the miR-7-5p binding sites or mutant EGFR 3' UTR with the mutated miR-7-5p binding sites were obtained from Sangon Biotech and inserted into the pmirGLO vectors (Promega, Madison, WI, USA) for the construction of reporters. The 293T cells were co-transfected with above the pmirGLO vectors and miR-7-5p mimic or NC mimic, respectively. After $48 \mathrm{~h}$ later, the luciferase activity was determined with the Dual-Luciferase Reporter Assay Kit (Promega).

\section{RT-qPCR analysis}

Reverse transcription-quantitative polymerase chain reaction (RT-qPCR) was performed by using $A B I$ PRISM 7900HT Fast Real-Time PCR System (Applied Biosystems, Foster City, CA) according to the 
manufacturer's instructions. Total RNA was isolated from cells and tumors with RNeasy Mini Kit (Qiagen, Valencia, CA). RNA quality was monitored and quantified by using the Qubit 2.0 Fluorometer (Invitrogen, Carlsbad, CA). The RNA samples were reverse transcribed into cDNA by using a cDNA Reverse Transcription Kit (Applied Biosystems, Foster City, CA). The cDNA (100 ng per sample) was subjected to qPCR analysis. The RT-qPCR cycling conditions consisted of $95^{\circ} \mathrm{C}$ for $3 \mathrm{~min}$; then 35 cycle amplification for $35 \mathrm{~s}$ at $95^{\circ} \mathrm{C}, 45 \mathrm{~s}$ at $58^{\circ} \mathrm{C}, 30 \mathrm{~s}$ at $72^{\circ} \mathrm{C}$; followed by $10 \mathrm{~min}$ at $72^{\circ} \mathrm{C}$. The primers used in this study were synthesized from Sangon Biotech (Shanghai, China). Primers sequences were as follows: RHPN1AS1 F: 5'-GCT CCT GGT CAT CAA GTT CCT CT-3', R: 5'-GCA CAG GCA CCA GAA TGA TCC-3'; miR-7-5p F: 5'-TGT TGT TTT GTG AT-3', R: 5'-GTG CAG GGT CCG AGG T-3'; U6 F: 5'-CAC TGG GTG CGG CAG GT-3', R: 5'-TCA TCA CCG ATC GAT ACG ATG A-3'; EGFR F: 5'- TGG TCA AGT GCT GGA TGA TAG A-3', R: 5'- ACG GTA GAA GTT GGA GTC TGT A-3'; GAPDH F: 5'- AAG TTC AAC GGC ACA GTC AAG G-3', R: 5'- CAT ACT CAG CAC CAG CAT CAC C -3 '. Analysis of the relative quantity gene expression was normalized by U6 and GAPDH expression and was performed by using the 2- $\Delta \Delta \mathrm{Ct}$ method.

\section{Western blot analysis}

Cells or tissues were lysed with lysis buffer $(150 \mathrm{mM} \mathrm{NaCl}, 1.0 \%$ NP-40 or $0.1 \%$ Triton X-100, $0.5 \%$ sodium deoxycholate, $0.1 \%$ SDS, $50 \mathrm{mM}$ Tris- $\mathrm{HCl}$ ( $\mathrm{pH} 8.0$ ), and protease inhibitors). After incubation on ice for 30 min, the homogenate was centrifuged at $14,000 \mathrm{rpm}$ for $30 \mathrm{~min}$ at $4{ }^{\circ} \mathrm{C}$, and the protein contents in the supernatant were quantified by BCA methods. Total protein was extracted from the supernatants, separated by $12 \%$ SDS-PAGE and transferred to PVDF membranes. The appropriate membranes were blocked by $5 \%$ BSA for $1 \mathrm{~h}$ at room temperature and incubated with primary antibodies, including antiEGFR (1:1000), anti-LC3- $\$ (1:1000), anti- LC3- $\$ (1:1000), anti-cleaved-caspase-3 (1:1000), anti-RARP (1:1000), anti-cleaved-RARP (1:1000), anti-Beclin-1 (1:1000), anti-Atg2 (1:1000), anti-p62 (1:1000), antiBax (1:1000), anti-Bcl-2 (1:1000), anti-PI3K (1:1000), anti-p-PI3K (1:1000), anti-AKT (1:1000), anti-p-AKT (1:1000), anti-mTOR (1:1000), anti-p-mTOR (1:1000)and anti- $\beta$-actin $(1: 1000)$ at $4{ }^{\circ} \mathrm{C}$ overnight. Then, antRabbit to mouse polyclonal secondary antibody (1:1000) was incubated for $2 \mathrm{~h}$ at room temperature. All immunoblots were visualized by using the ChemiDoc MP System (Bio-Rad protein assay; Bio-Rad, Segrate, Italy) and the enhanced chemiluminescence reagents (Pierce, Rockford, IL, USA). The protein levels were quantitated by Quantity One software (Bio-Rad Laboratories, Hercules, CA).

\section{Immunofluorescence}

Cells were grown on glass coverslips to a confluence of 30-50\%. Then, they were transfected with shRHPN1-AS1 for 6, 24 and $48 \mathrm{~h}$, washed twice with PBS, and fixed for $10 \mathrm{~min}$ in $4 \%$ paraformaldehyde. Cells were permeabilized in $0.5 \%$ Triton X-100, washed, and blocked for $2 \mathrm{~h}$ in $5 \%$ normal serum. After blocking, the cells were incubated with anti-LC3 (1:500) for $24 \mathrm{~h}$ at $4{ }^{\circ} \mathrm{C}$, washed with PBS and incubated with fluorescently labeled secondary antibody $(1: 500)$ and DAPI $(5 \mu \mathrm{g} / \mathrm{mL})$ for $2 \mathrm{~h}$ at room temperature. Finally, coverslips were washed and mounted with Vectashield (Solarbio). Immunofluorescence staining was imaged using a fluorescence microscope (Olympus). Five representative fields were captured at $x$ 
200 magnification, and the numbers of cells expressing target proteins in the cytoplasm and the nucleus (overlapping with DAPI) cells were counted.

\section{Transmission electron microscopy (ETM)}

PC3 cells were harvested after transfection with miR-7-5p mimic or NC mimic, fixed with ice-cold $2.5 \%$ glutaraldehyde in PBS for $2 \mathrm{~h}$, post-fixed in 1\% osmium tetroxide, dehydrated in a graded series of ethanol (50-100\%) and acetone, and embedded in Epon. Representative areas were chosen for ultrathin sectioning and examined with a transmission electron microscope.

\section{Vital staining with acridine orange (AO) or monodansylcadaverine (MDC) staining}

PCa cells were seeded in a 24-well culture plate and after subsequent treatments, cells were stained with AO $(1 \mu \mathrm{g} / \mathrm{mL})$ or MDC $(0.05 \mathrm{mmol} / \mathrm{L})$ directly for $10 \mathrm{~min}$ at $37^{\circ} \mathrm{C}$, and then observed under the fluorescence microscope (Olympus, Tokyo, Japan).

\section{Transwell assay}

PC3 cells in the logarithmic growth phase were digested and counted, and were cultured in the upper transwell chamber, with $5 \times 10^{4}$ cells/well and $200 \mu \mathrm{L}$ serum-free medium. Subsequently, $500 \mu \mathrm{L}$ DMEM containing $10 \% \mathrm{FBS}$ was added to the lower chamber. After incubation at $37^{\circ} \mathrm{C}$ with $5 \% \mathrm{CO}_{2}$ for $24 \mathrm{~h}$, the upper chamber cells were carefully removed. And the lower membrane cells were fixed in formaldehyde for $15 \mathrm{~min}$, and then stained with crystal violet (Beyotime, China) for $20 \mathrm{~min}$. Ultimately, 10 fields were randomly selected by using an optical microscope (Olympus, Tokyo, Japan).

\section{Flow Cytometry analysis}

PC3 and LNCaP cells after transfection were digested, collected, and re-suspended in Binding Buffer, and then added with $5 \mu \mathrm{L}$ Annexin V-FITC regent and $10 \mu \mathrm{L}$ propidium iodide (PI) regent, mixed gently, and placed in the dark at room temperature for $15 \mathrm{~min}$. After washing in PBS, apoptotic cells were analyzed by flow cytometry (BD FACSCalibur cytometer, Becton Dickinson, San Jose, CA, USA) using an Annexin VFITC apoptosis detection kit (Beyotime Biotechnology, China) following the manufacturer's instructions.

\section{Cell counting kit-8 (CCK-8) assay}

PC3 cell viability was examined by using a Cell Counting Kit-8 (CCK-8, Dojindo, JPN) following the user's

guide. $1 \times 10^{3}$ cells/well after transfection were harvested and seeded into 96 -well plates). After that, cells were cultured and processed with $10 \mu \mathrm{L}$ of CCK-8 solution for $12,24,36,48$ or $72 \mathrm{~h}$. Finally, absorbance at $450 \mathrm{~nm}$ was examined by using a microplate reader (Olympus, Tokyo, Japan).

\section{Statistical analysis}

The values are shown as the means \pm SEM for triplicate experiments, and significant differences were calculated using the ANOVA test and Student's t-test. Levels of statistical significance were set at $P<0.05$ 
or 0.01 as indicated.

\section{Results}

\section{RHPN1-AS1 is up-regulated in prostate cancer, and silencing RHPN1-AS1 inhibits prostate cancer cell proliferation, invasion, and induces apoptosis.}

According to the statistics data collected from the GEPIA database, we found that PCa patients with high RHPN1-AS1 expression had a shorter survival time (Fig. 1A), which indicated that high expression of RHPN1-AS1 was highly associated with poor prognosis of PCa patients. Moreover, twenty-one PCa patients were recruited in this study, and RHPN1-AS1 expression was detected in tissue samples collected from these patients. After RT-qPCR exanimation, we determined that RHPN1-AS1 was expressed higher in tumor tissue samples compared with adjacent normal tissues (Fig. 1B). Consistently, RHPN1-AS1 expressed at a higher level in PCa cell lines compared with normal epithelial cell PWPE-1 (Fig. 1C). Furthermore, we also observed that proliferation and invasiveness were significantly inhibited and apoptosis was markedly induced in PC3 cells that were transfected with sh-RHPN1-AS1 (Fig. 1D-F). These data suggested that RHPN1-AS1 potentially participated in tumorigenesis of PCa.

\section{Silencing RHPN1-AS1 induces G2/M cell cycle arrest and switches apoptosis and autophagy in prostate cancer cells.}

To investigate the regulatory mechanism of RHPN1-AS1 in PCa cells, we employed flow cytometry to assess whether RHPN1-AS1 affected cell cycle progression and apoptosis. Silencing RHPN1-AS1 induced the proportion of cells in the G2/M phase and cells in the sub G1 phase were significantly increase in PC3 cells (Fig. 2A), which indicated that apoptosis was inhibited by RHPN1-AS1. An annexin V-PE/7-AAD assay further confirmed that silencing RHPN1-AS1 did not significantly induce apoptosis in PC3 cells at 12 and $24 \mathrm{~h}$, but significantly induced PC3 apoptosis at $48 \mathrm{~h}$ (Fig. 2B and C).

The multiple layers of interaction between the processes of autophagy and apoptosis present, which is a seamless balance between life and death in response to given cellular stress[18]. Therefore, we verified whether apoptosis induced by silencing RHPN1-AS1 in PC3 cells was related to autophagy. The results showed that after $12 \mathrm{~h}$ of silencing RHPN1-AS1, PC3 cells exhibited a stark increase in discrete acidic vesicles, especially at 24 and $48 \mathrm{~h}$, at which greater than $70 \%$ of the cells were MDC-positive, and an average of more than $15 \mathrm{AO}$ puncta was dispersed throughout the cytoplasm in PC3 cells with silencing RHPN1-AS1 (Fig. 2D and E). Next, autophagy activity was further validated by silencing RHPN1-AS1 induced LC3 puncta in PC3 cells with immunofluorescence microscopy (Fig. 2F and G). All in all, based on the above results, we demonstrated that silencing RHPN1-AS1 simultaneously induced G2/M cell cycle arrest, autophagy and apoptosis. Furthermore, autophagy precedes apoptosis in RHPN1AS1silenced PC3 cells, but the relationship between silencing RHPN1-AS1 induced autophagy and apoptosis needs to be further investigated. 
Silencing RHPN1-AS1 induces autophagy contributes to caspase-dependent apoptosis in prostate cancer cells.

There is a complex interplay between autophagy and apoptosis that is highly dependent on the cellular environment or the stimulus. To investigate the relationship between autophagy and apoptosis induced by silencing RHPN1-AS1, a vitality assay was performed with PC3 cells pretreated with 5 mM 3MA, $20 \mu \mathrm{m}$ z-VAD-fmk or vehicle alone before silencing RHPN1-AS1. Treatment with 3MA significantly reduced silencing RHPN1-AS1 induced AO puncta and LC3-I to LC3-II conversion (Fig. 3C and E). Moreover, the inhibition of autophagy by 3MA antagonised the promoting effects of RHPN1-AS1 on PC3 cell viability (Fig. 3A), thus suggesting that, at least in PC3 cells, silencing RHPN1-AS1 activated cytotoxic autophagy, which can be inhibited to restore cell viability. To further demonstrate whether silencing RHPN1-AS1 triggered autophagy inhibits or promotes apoptosis, PC3 cells in which autophagy was suppressed by 3MA with silencing or not silencing RHPN1-AS1 underwent a flow cytometry apoptosis assay. The results showed that 3MA-mediated autophagy inhibition moderately eliminated silencing RHPN1-AS1 induced apoptosis, which was consistent with the reduced cleaved-caspase-3 and cleaved-PARP (Fig. 3E).

Subsequently, the effect of inhibition of apoptosis on autophagy was determined. We observed that zVAD-fmk had a minimal effect on the number of AO-staining red acidic vesicles induced by silencing RHPN1-AS1, in accordance with the expression of LC3-II (Fig. 3B and E). However, after pretreatment with z-VAD-fmk, a portion of the cell vitality was successfully restored and apoptosis was nearly abrogated in RHPN1-AS1 silenced cells (Fig. 3A, C and D). Downregulated cleaved-caspase-3 and cleaved-PARP further demonstrated that z-VAD-fmk completely relieved silencing RHPN1-AS1 mediated apoptosis (Fig. 3F). Those indicated that silencing RHPN1-AS1 induced apoptosis is caspase-dependent.

\section{RHPN1-AS1 binds to miR-7-5p and downregulates miR-7-5p expression.}

To further investigate the regulatory mechanism of RHPN1-AS1 in PCa cells, the subcellular localization of RHPN1-AS1 was detected. The results revealed that RHPN1-AS1 was positioned on the cytoplasm rather than the nucleus in PC3 cells (Fig. 4A). This finding suggested that RHPN1-AS1 exerts its downstream effects by post-transcriptional epigenetic regulation. By conducting starBase v3.0 (http://starbase.sysu.edu.cn/index.php), we found that miR-7-5p could bind with RHPN1-AS1 (Fig. 4B). To verify the binding capacity between RHPN1-AS1 and miR-7-5p, a luciferase reporter gene assay was carried out and found that miR-7-5p could inhibit the luciferase activity of wild-type RHPN1-AS1 vectors (Fig. 4C). Furthermore, miR-7-5p was significantly upregulated in PCa tissues, and overexpression of RHPN1-AS1 inhibited miR-7-5p expression in PC3 cells (Fig. 4D-F). These results suggested that RHPN1AS1 bound with miR-7-5p and inhibited miR-7-5p expression, thereby regulating PCa cells.

\section{Overexpression of miR-7-5p inhibits prostate cancer cell proliferation and promotes autophagy and apoptosis.}

To explain the role of miR-7-5p in PCa cells autophagy and apoptosis, we transfected PC3 cells with miR7-5p mimic or NC mimic, and then observed the effects of miR-7-5p on PC3 cells. The results showed that 
miR-7-5p expression levels were markedly upregulated in PC3 cells transfected with miR-7-5p mimic (Fig. 5A). Moreover, we found that overexpression of miR-7-5p significantly inhibited the proliferation and increased the number of apoptotic cells in PC3 cells (Fig. 5B-D). To further demonstrate whether miR-7-5p regulates autophagy in PC3 cells underwent TEM to observe the ultrastructure of PC3 cells after transfection with miR-7-5p mimic or NC mimic. Higher magnification of autophagosomes revealed a smooth double-membrane containing cellular material, and intense electron-dense lysosomal structures were clearly observed in miR-7-5p overexpressed PC3 cells compared to cells transfected with NC mimic (Fig. 5E). Therefore, our data revealed that miR-7-5p could inhibit cell proliferation and promote autophagy and apoptosis of PC3 cells to participate PCa progression.

\section{EGFR is a target of miR-7-5p and is regulated by miR-7-5p.}

Biological prediction website starBase v3.0 (http://starbase.sysu.edu.cn/index.php) was used to predict the potential target genes of miR-7-5p. The prediction results identified that there was a specific binding region between the 3'UTR of EGFR and miR-7-5p sequences, indicating EGFR may be a target gene of miR-7-5p (Fig. 6A). The dual-luciferase reporter gene assay was performed to verify the binding relationship between EGFR and miR-7-5p. The results showed that the luciferase activity of the EGFR wild type was significantly inhibited by miR-7-5p mimic, but the luciferase activity of the EGFR mutant was not inhibited (Fig. 6B). Then, we overexpressed miR-7-5p or inhibited miR-7-5p in PC3 cells, and the results showed that overexpression of miR-7-5p promoted expression of miR-7-5 and inhibited EGFR expression (Fig. 6C and D). Subsequently, we overexpressed RHPN1-AS1 and/or miR-7-5p in PC3 cells, and the results showed that overexpression of RHPN1-AS1 promoted EGFR expression, whereas miR-7-5p overexpression reversed the promoting effect of RHPN1-AS1 on EGFR expression (Fig. 6E). The above results indicated that RHPN1-AS1 acted as a ceRNA of miR-7-5p to upregulate EGFR expression.

\section{Silencing EGFR counteracts the inhibitory effect of RHPN1-AS1 on prostate cancer cell apoptosis.}

To further determine whether RHPN1-AS1 inhibited apoptosis and autophagy in PC3 cells through upregulating EGFR expression, we performed overexpression of RHPN1-AS1 and/or silencing EGFR in PC3 cells and observed that overexpression of RHPN1-AS1 increased the expression levels of RHPN1-AS1 and EGFR (Fig. 8A, B and C). Moreover, we found that overexpression of RHPN1-AS1 decreased the expression of autophagy relative protein Beclin-1 and Atg12, increased p62 expression, and reduced LC3-I to LC3-II conversion, silencing EGFR reversed the effects of RHPN1-AS1 on autophagy relative protein expression (Fig. 7D and E). Then, we detected the apoptotic relative protein expression and cell apoptosis in PC 3cells transfected with RHPN1-AS1 and/or si-EGFR. The results showed that apoptotic relative protein cleaved-caspase-3, cleaved-PARP and Bax expression were markedly reduced, and Bcl-2 expression was increased in RHPN1-AS1 overexpressed PC3 cells. Silencing EGFR promoted the protein expression of cleaved-caspase-3, cleaved-PARP and Bax and inhibited Bcl-2 protein expression, while the apoptotic relative protein expression returned to control levels in PC3 cells co-transfected with RHPN1AS1 and si-EGFR (Fig. 7F and G). In addition, we observed that overexpression of RHPN1-AS1 inhibited cell apoptosis, and silencing EGFR induced apoptosis in PC3 cells (Fig. 7H and I). From the results above, 
it could be concluded that silencing RHPN1-AS1 induced autophagy and apoptosis in PCa cells through upregulating EGFR expression.

The PI3K/AKT-mTOR pathway is downstream of the EGFR gene and plays a crucial role in regulating the progression of autophagy. Here, we found that the phosphorylation levels of PI3K and AKT involved in autophagy were obviously upregulated in RHPN1-AS1 overexpressed PC3 cells and markedly reduced in silencing EGFR PC3 cells (Fig. 7J). These results uncovered that overexpression of RHPN1-AS1 activated the EGFR/AKT/mTOR pathway.

\section{RHPN1-AS1 inhibits prostate cancer cell apoptosis through the PI3K/AKT/mTOR pathway.}

To further determine whether the PI3K/AKT/mTOR pathway was involved in cell apoptosis induced by RHPN1-AS1 silencing, we pretreated PC3 and LNCaP cells with GSK2126458 (a PI3K inhibitor) before overexpression of RHPN1-AS1. The results showed that expression of RHPN1-AS1 and EGFR was markedly upregulated and miR-7-5p expression was decreased in RHPN1-AS1 overexpressed PC3 and LNCaP cells, While GSK2126458 had no effect on expression of RHPN1-AS1, miR-7-5p and EGFR (Fig. 8AC). Next, we observed that overexpression of RHPN1-AS1 promoted the phosphorylation of the $\mathrm{PI} 3 \mathrm{~K} / \mathrm{AKT} / \mathrm{mTOR}$ pathway key protein PI3K, AKT and mTOR, inhibited autophagy relative protein Beclin-1 expression, and reduced LC3-I to LC3-II conversion in PC3 and LNCaP cells by using Western blotting (Fig. 8D and E). Furthermore, we also found that the number of $A O$ puncta formation in PC3 and LNCaP cells were significantly reduced by overexpressing RHPN1-AS1, and GSK2126458 counteracted the effects of RHPN1-AS1 on AO puncta formation (Fig. 8F). Then, we examined the apoptosis of PC3 and LNCaP cells by using Western blot and flow cytometry, and found that overexpression of RHPN1-AS1 significantly decreased the expression of cleaved-caspase-3, a protein involved in apoptosis, and reduced the number of apoptosis cells in PC3 and LNCaP cells, which was reversed by GSK2126458 (Fig. 8D, G and $H$ ). Hence, these data suggested that silencing RHPN1-AS1 suppressed PCa tumor progression by inducing PCa cell apoptosis through the PI3K/AKT/mTOR pathway.

\section{Discussion}

Despite the progressive decline in the incidence and mortality of prostate cancer ( $\mathrm{PCa})$, it is still considered the most common urological malignancy afflicting the male population worldwide[3,5]. Currently, accumulating evidence suggests that it is possible to develop new therapies for the treatment of PCa. The mTOR signaling pathway is a pivotal regulator in PCa cells and may function as an inhibitor of PCa cells when inhibited in clinical trials. The PI3K/AKTmTOR pathway is important in regulating cell survival signals, and its full inhibitory function has been suggested as a possible therapeutic mechanism for the treatment of PCa[19, 21]. This study aimed to investigate the role of IncRNA RHPN1-AS1 in the regulation of apoptosis and autophagy in PCa cells through EGFR mediated the PI3K/AKTmTOR signaling pathway.

An increasing number of studies have shown that IncRNAs are important factors in human diseases and ontogeny. Aberrant expression of IncRNAs may lead to tumorigenesis and aggressive progression, 
making these molecules attractive therapeutic targets[22]. A key finding of a previous study was that RHPN1-AS1 was upregulated in PCa tissues[9]. In our current study, we investigated whether RHPN1-AS1 is a regulator of $\mathrm{PC}$ a tumorigenesis. Through a loss of function experiments, we determined that RHPN1AS1 has a positive effect on PCa cell proliferation but a negative effect on PCa cell apoptosis. Therefore, the present study determined the oncogenic properties of RHPN1-AS1 in PCa tumorigenesis.

Related studies have revealed the interaction between IncRNAs, miRNAs and target genes in the mechanism study of tumorigenesis, and IncRNAs can function by acting as ceRNA of miRNAs to regulate downstream target genes[23]. For example, You et al. found that DSCR8 competitively binds to Yin Yang 1 (YY1) with miR-3192-5p to promote the proliferation and migration and inhibit apoptosis in ovarian cancer cells, thereby promoting ovarian cancer progression[24]. Fen et al. found that RHPN1-AS1 reduced miR-596 expression by sponging it and enhanced insulin-like growth factor 2 mRNA-binding protein 2 (IGF2BP2) expression to accelerate proliferation and metastasis of hepatocellular carcinoma cells[15]. In our study, we found that RHPN1-AS1 acted as a ceRNA of miR-7- $5 p$ to upregulate EGFR in PCa cells, which was consistent with previous reports $[13,25]$.

It is well known that aberrant proliferation and cell apoptosis are one of the hallmarks of cancer cells, and inhibition of proliferation or induction of cell apoptosis is a key to anti-tumor research[26]. Previous studies have found that the expression of RHPN1-AS1 in epithelial ovarian cancer (EOC) tissues were higher than that in para-cancerous control tissues. Overexpression of RHPN1-AS1 promoted proliferation and metastasis of EOC cells, knockdown of RHPN1-AS1 significantly inhibited the proliferation, migration and invasion of ovarian cancer cells[10, 27]. In retinoblastoma (RB) cells, RHPN1-AS1 was upregulated. Silencing RHPN1-AS1 inhibited the activity of RB cells and promoted apoptosis[28]. In our study, we found that expression of RHPN1-AS1 markedly upregulated in PCa tissues and cells, silencing RHPN1AS1 inhibited proliferation and induced apoptosis in PCa cells. Moreover, we also found that overexpression of miR-7-5p or silencing EGFR induced PCa cell apoptosis. From this, we determined that RHPN1-AS1 promoted proliferation and invasion and inhibited apoptosis in PCa cells through acting as a ceRNA of miR-7- $5 p$ to upregulate EGFR.

In some cellular contexts, autophagy acts as a cell survival pathway that prevents tumor cells from inducing apoptosis in response to cancer therapy, whereas in other settings, prolonged stress and progressive autophagy may ultimately lead to cell death, either in concert with apoptosis, or as a backup mechanism[17, 29]. TEM revealed that the accumulation of AO puncta, the formation of autophagosomes, and the increased LC3-I/LC3-II conversion all induced autophagy[30]. We further studied the effect of RHPN1-AS1 on the biological function of PCa cells, and on the basis of this study, we found that silencing RHPN1-AS1 induced apoptosis and autophagy in PCa cells. However, RHPN1AS1 inhibition induced cell death was markedly restored by 3MA or Z-VAD-fmk, indicating that autophagic cell death induced by RHPN1-AS1 inhibition is a cause of apoptosis and is caspase dependent. Recently, cytotoxic autophagy was also observed in cervical cancer ceels[31]. To better understand the underlying mechanism by which RHPN1-AS1 inhibition induces autophagy, we explored the downstream pathways. 
EGFR is the key mediator of cell proliferation, differentiation and transformation. Transactivation of EGFR is of great significance for tumor cell growth[32, 33]. EGFR ligand binding promotes receptor dimerization, autophosphorylation and subsequent activation of downstream PI3K/AKT and RAS/MAPK/ERK signaling pathways to regulate tumor cell growth[34,35]. Previous studies have established that the $\mathrm{PI} 3 \mathrm{~K} / \mathrm{AKT} / \mathrm{mTOR}$ pathway as a key regulator of autophagy, is involved in the initiation and promotion of a range of pathological diseases, including many human cancers[19, 36]. Inhibition of the PI3K/AKTmTOR pathway is important for suppressing tumor progression in $\mathrm{PCa}[33,37]$, as demonstrated in our study, silencing RHPN1-AS1 inhibited phosphorylation of PI3K, AKT and mTOR and induced autophagy and apoptosis in PCa cells, which is reversed by GSK2126458 (a PI3K inhibitor). This also suggested that silencing RHPN1-AS1 induces apoptosis and autophagy in PCa cells via suppressing the PI3K/AKTmTOR pathway.

In summary, RHPN1-AS1 functions as an oncogenic property IncRNA in PCa tumorigenesis, and RHPN1AS1 inhibition induces autophagy and apoptosis in PCa cells through the miR-7-

5p/EGFR/PI3K/AKT/mTOR signaling pathway. Our founding provides ideas and an experimental basis for PCa improvement and treatment.

\section{Declarations}

\section{Acknowledgement}

We appreciate all members involved in this study.

\section{Author contributions}

MXL and MHB has conceived and designed the work. RHT, ZY and WBF performed all the experiments and analyzed data. MXL developed the first draft of the manuscript and reviewed by RHT, ZY, WBF and MHB. All authors read the manuscript for adequate correction and approved the final copy of it.

\section{Compliance with ethical standards}

Conflict of interest There are no conflicts of interest of any kind in this study.

Ethical approval This study was approved by the Ethics Committees of the Second Affiliated Hospital of Xi'an Jiaotong University.

Consent to participate Each participant signed written informed consent before the study.

Consent for publication All authors consent to publish this work.

\section{References}


1. Brandão A, Paulo PTeixeira MR (2020) Hereditary Predisposition to Prostate Cancer: From Genetics to Clinical Implications. Int J Mol Sci 21(14).http://dx.doi.org/10.3390/ijms21145036

2. Wilt TJ, Ullman KE, Linskens EJ, MacDonald R, Brasure M, Ester E, Nelson VA, Saha J, Sultan SDahm P (2021) Therapies for Clinically Localized Prostate Cancer: A Comparative Effectiveness Review. J Urol 205(4): 967-976.http://dx.doi.org/10.1097/ju.0000000000001578

3. Siegel DA, O'Neil ME, Richards TB, Dowling NFWeir HK (2020) Prostate Cancer Incidence and Survival, by Stage and Race/Ethnicity - United States, 2001-2017. MMWR Morb Mortal Wkly Rep 69(41): 1473-1480.http://dx.doi.org/10.15585/mmwr.mm6941a1

4. Remmers S, Roobol MJ (2020) Personalized strategies in population screening for prostate cancer. Int J Cancer 147(11): 2977-2987.http://dx.doi.org/10.1002/ijc.33045

5. Carlsson SV, Vickers AJ (2020) Screening for Prostate Cancer. Med Clin North Am 104(6): 10511062.http://dx.doi.org/10.1016/j.mcna.2020.08.007

6. Zhang E, Zhang M, Shi C, Sun L, Shan L, Zhang HSong Y (2020) An overview of advances in multiomics analysis in prostate cancer. Life Sci 260: 118376.http://dx.doi.org/10.1016/j.lfs.2020.118376

7. Taniue K, Akimitsu N (2021) The Functions and Unique Features of LncRNAs in Cancer Development and Tumorigenesis. Int J Mol Sci 22(2).http://dx.doi.org/10.3390/ijms22020632

8. Statello L, Guo CJ, Chen LLHuarte M (2021) Gene regulation by long non-coding RNAs and its biological functions. Nat Rev Mol Cell Biol 22(2): 96-118.http://dx.doi.org/10.1038/s41580-02000315-9

9. Cai J, Chen Z, Chen X, Huang H, Lin XMiao B (2020) Coexpression Network Analysis Identifies a Novel Nine-RNA Signature to Improve Prognostic Prediction for Prostate Cancer Patients. Biomed Res Int 2020: 4264291.http://dx.doi.org/10.1155/2020/4264291

10. Wang J, Ding W, Xu Y, Tao E, Mo M, Xu W, Cai X, Chen X, Yuan JWu X (2020) Long non-coding RNA RHPN1-AS1 promotes tumorigenesis and metastasis of ovarian cancer by acting as a ceRNA against miR-596 and upregulating LETM1. Aging (Albany NY) 12(5): 4558-

4572.http://dx.doi.org/10.18632/aging.102911

11. Ma L, Li T, Liu G, Wang J, Yin ZKang J (2020) LncRNA RHPN1-AS1 modulates cholangiocarcinoma progression and is related with poor clinical outcomes through miR-345-5p/YAP1 axis. J Gastroenterol Hepatol.http://dx.doi.org/10.1111/jgh.15374

12. Duan H, Li X, Chen Y, Wang YLi Z (2019) LncRNA RHPN1-AS1 promoted cell proliferation, invasion and migration in cervical cancer via the modulation of miR-299-3p/FGF2 axis. Life Sci 239: 116856.http://dx.doi.org/10.1016/j.lfs.2019.116856

13. Zheng W, Li H, Zhang H, Zhang C, Zhu Z, Liang HZhou Y (2020) Long noncoding RNA RHPN1-AS1 promotes colorectal cancer progression via targeting miR-7-5p/OGT axis. Cancer Cell Int 20: 54.http://dx.doi.org/10.1186/s12935-020-1110-9

14. Ding L, Wang L, Li Z, Jiang X, Xu YHan N (2020) The positive feedback loop of RHPN1-AS1/miR1299/ETS1 accelerates the deterioration of gastric cancer. Biomed Pharmacother 124: 109848.http://dx.doi.org/10.1016/j.biopha.2020.109848 
15. Fen H, Hongmin Z, Wei W, Chao Y, Yang Y, Bei LZhihua S (2020) RHPN1-AS1 Drives the Progression of Hepatocellular Carcinoma via Regulating miR-596/IGF2BP2 Axis. Curr Pharm Des 25(43): 46304640.http://dx.doi.org/10.2174/1381612825666191105104549

16. Levine B, Kroemer G (2019) Biological Functions of Autophagy Genes: A Disease Perspective. Cell 176(1-2): 11-42.http://dx.doi.org/10.1016/j.cell.2018.09.048

17. Onorati AV, Dyczynski M, Ojha RAmaravadi RK (2018) Targeting autophagy in cancer. Cancer 124(16): 3307-3318.http://dx.doi.org/10.1002/cncr.31335

18. D'Arcy MS (2019) Cell death: a review of the major forms of apoptosis, necrosis and autophagy. Cell Biol Int 43(6): 582-592.http://dx.doi.org/10.1002/cbin.11137

19. Xu Z, Han X, Ou D, Liu T, Li Z, Jiang G, Liu JZhang J (2020) Targeting PI3K/AKT/mTOR-mediated autophagy for tumor therapy. Appl Microbiol Biotechnol 104(2): 575-

587.http://dx.doi.org/10.1007/s00253-019-10257-8

20. Braglia L, Zavatti M, Vinceti M, Martelli AMMarmiroli S (2020) Deregulated PTEN/PI3K/AKT/mTOR signaling in prostate cancer: Still a potential druggable target? Biochim Biophys Acta Mol Cell Res 1867(9): 118731.http://dx.doi.org/10.1016/j.bbamcr.2020.118731

21. Shorning BY, Dass MS, Smalley MJPearson HB (2020) The PI3K-AKT-mTOR Pathway and Prostate Cancer: At the Crossroads of AR, MAPK, and WNT Signaling. Int J Mol Sci 21(12).http://dx.doi.org/10.3390/ijms21124507

22. Peng WX, Koirala PMo YY (2017) LncRNA-mediated regulation of cell signaling in cancer. Oncogene 36(41): 5661-5667.http://dx.doi.org/10.1038/onc.2017.184

23. Chan JJ, Tay Y (2018) Noncoding RNA:RNA Regulatory Networks in Cancer. Int J Mol Sci 19(5).http://dx.doi.org/10.3390/ijms19051310

24. You Q, Yao Y, Wu J, Cheng C, Li YYuan H (2020) YY1-induced IncRNA DSCR8 promotes the progression of ovarian cancer via miR-3192-5p/YY1 axis. Biomed Pharmacother 129:

110339.http://dx.doi.org/10.1016/j.biopha.2020.110339

25. Song XZ, Ren XN, Xu XJ, Ruan XX, Wang YLYao TT (2020) LncRNA RHPN1-AS1 Promotes Cell Proliferation, Migration and Invasion Through Targeting miR-7-5p and Activating PI3K/AKT/mTOR Pathway in Hepatocellular Carcinoma. Technol Cancer Res Treat 19: 1533033820957023.http://dx.doi.org/10.1177/1533033820957023

26. Varghese E, Samuel SM, Sadiq Z, Kubatka P, Liskova A, Benacka J, Pazinka P, Kruzliak PBüsselberg D (2019) Anti-Cancer Agents in Proliferation and Cell Death: The Calcium Connection. Int J Mol Sci 20(12).http://dx.doi.org/10.3390/ijms20123017

27. Zhao L, Liu T, Zhang X, Zuo DLiu C (2020) IncRNA RHPN1-AS1 Promotes Ovarian Cancer Growth and Invasiveness Through Inhibiting miR-1299. Onco Targets Ther 13: 5337-

5344.http://dx.doi.org/10.2147/ott.s248050

28. Li ZN, Ge MX, Cao LJYuan ZF (2020) IncRNA RHPN1-AS1 Serves as a Sponge for miR-3133 Modulating the Cell Proliferation of Retinoblastoma through JAK2. Biomed Res Int 2020: 3502981.http://dx.doi.org/10.1155/2020/3502981 
29. Galluzzi L, Green DR (2019) Autophagy-Independent Functions of the Autophagy Machinery. Cell 177(7): 1682-1699.http://dx.doi.org/10.1016/j.cell.2019.05.026

30. Kuma A, Komatsu MMizushima N (2017) Autophagy-monitoring and autophagy-deficient mice. Autophagy 13(10): 1619-1628.http://dx.doi.org/10.1080/15548627.2017.1343770

31. Yang Y, Wang Q, Song D, Zen R, Zhang L, Wang Y, Yang H, Zhang D, Jia J, Zhang JWang J (2020) Lysosomal dysfunction and autophagy blockade contribute to autophagy-related cancer suppressing peptide-induced cytotoxic death of cervical cancer cells through the AMPK/mTOR pathway. J Exp Clin Cancer Res 39(1): 197.http://dx.doi.org/10.1186/s13046-020-01701-z

32. Sigismund S, Avanzato DLanzetti L (2018) Emerging functions of the EGFR in cancer. Mol Oncol 12(1): 3-20.http://dx.doi.org/10.1002/1878-0261.12155

33. Gazzeri S (2018) [Nuclear EGFR: a new mode of oncogenic signalling in cancer]. Biol Aujourdhui 212(1-2): 27-33.http://dx.doi.org/10.1051/jbio/2018016

34. Xu MJ, Johnson DEGrandis JR (2017) EGFR-targeted therapies in the post-genomic era. Cancer Metastasis Rev 36(3): 463-473.http://dx.doi.org/10.1007/s10555-017-9687-8

35. Dai N, Ye R, He Q, Guo P, Chen HZhang Q (2018) Capsaicin and sorafenib combination treatment exerts synergistic anti-hepatocellular carcinoma activity by suppressing EGFR and PI3K/Akt/mTOR signaling. Oncol Rep 40(6): 3235-3248.http://dx.doi.org/10.3892/or.2018.6754

36. Wang Y, Zhang H (2019) Regulation of Autophagy by mTOR Signaling Pathway. Adv Exp Med Biol 1206: 67-83.http://dx.doi.org/10.1007/978-981-15-0602-4_3

37. Tiemin P, Fanzheng M, Peng X, Jihua H, Ruipeng S, Yaliang L, Yan W, Junlin X, Qingfu L, Zhefeng H, Jian L, Zihao G, Guoxing L, Boshi S, Ming Z, Qinghui M, Desen LLianxin L (2020) MUC13 promotes intrahepatic cholangiocarcinoma progression via EGFR/PI3K/AKT pathways. J Hepatol 72(4): 761773.http://dx.doi.org/10.1016/j.jhep.2019.11.021

\section{Figures}


A

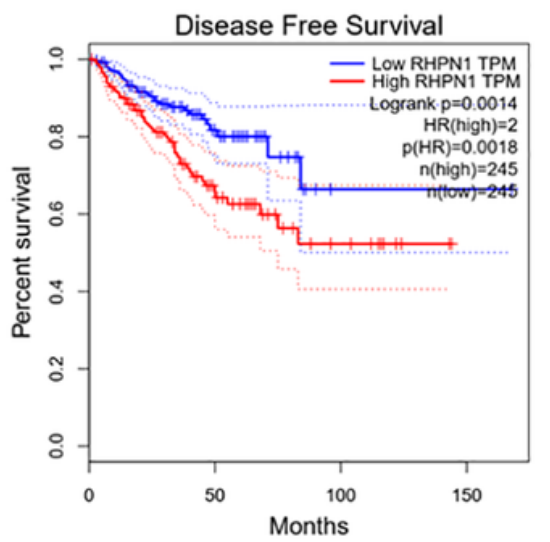

D

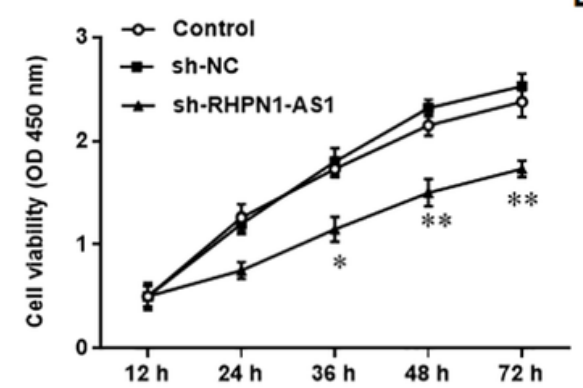

F

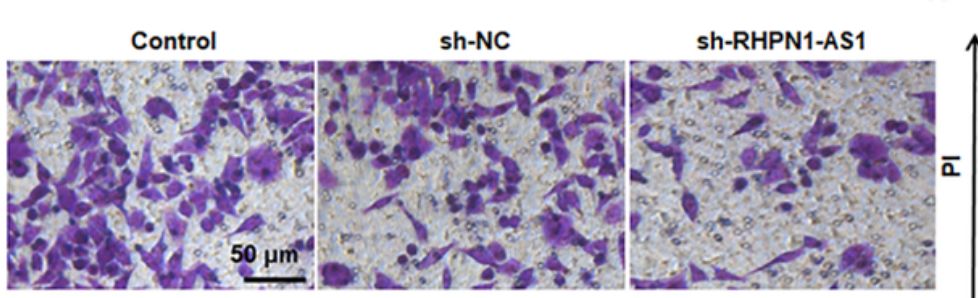

B

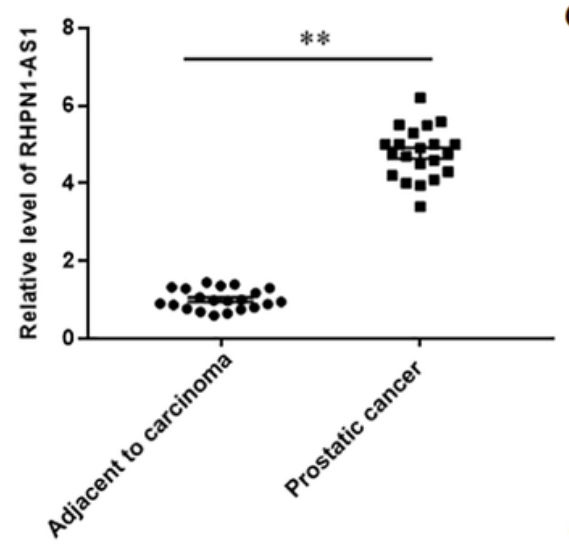

E

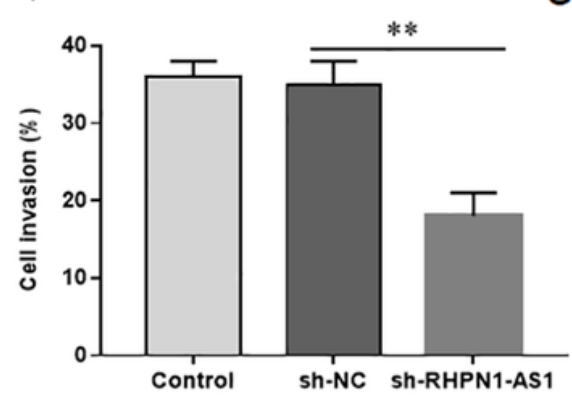

H
C

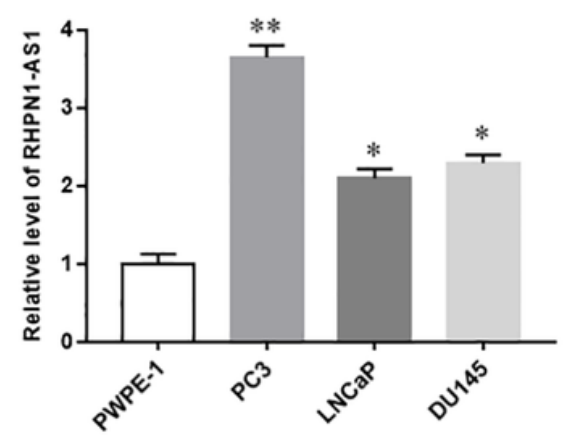

G

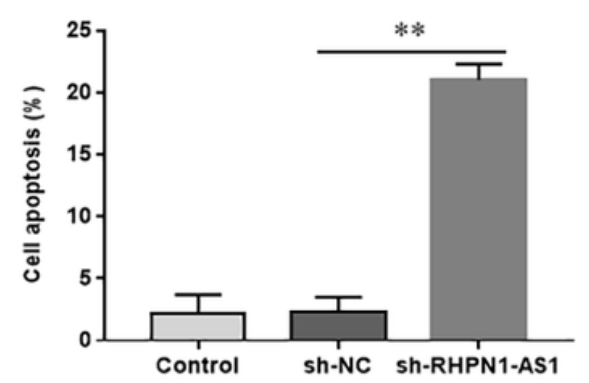

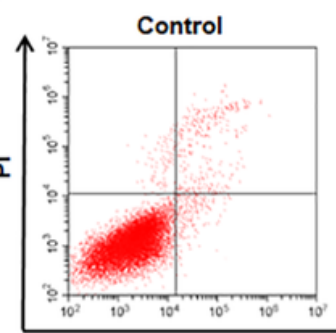

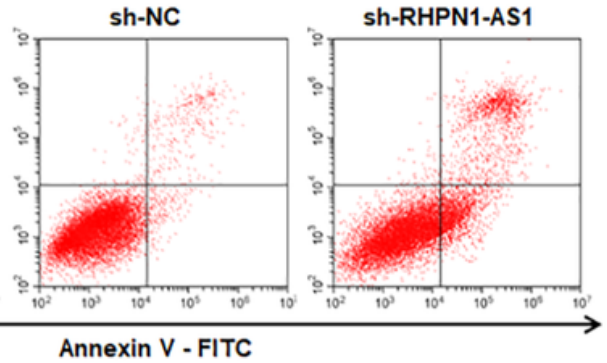

\section{Figure 1}

Expression of RHPN1-AS1 in prostate cancer and effects on prostate cancer cells. (A) The effect of RHPN1-AS1 expression level on prostate cancer patient survival in the TCGA database. (B) The expression of RHPN1-AS1 was detected by RT-qPCR in prostate cancer tissues and adjacent normal tissues, $\mathrm{N}=21$. (C) The expression of RHPN1-AS1 was detected by RT-qPCR in PWPE-1, PC3, LNCaP, and DU145 cells, N=5. PC3 cells were transfected with sh-RHPN1-AS1 or sh-NC for $48 \mathrm{~h}$. (D) The proliferation ability of PC3 cells was measured by CCK-8 assay, N=5. (E and F) The invasion abilities of PC3 cells were detected by Transwell assay, N=4. (G and H) PC3 cell apoptosis was detected with flow cytometry assay, $\mathrm{N}=4$. The difference of the samples between the two groups were analyzed with independent sample $t-$ test, and the analysis of variance test was used to evaluate multigroup comparisons of the means. Compared with PWPE-1 cells or control group, *P $<0.05$, ** $\mathrm{P}<0.01$. 
A

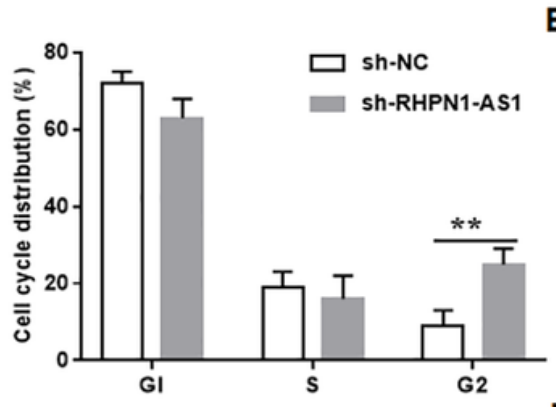

C

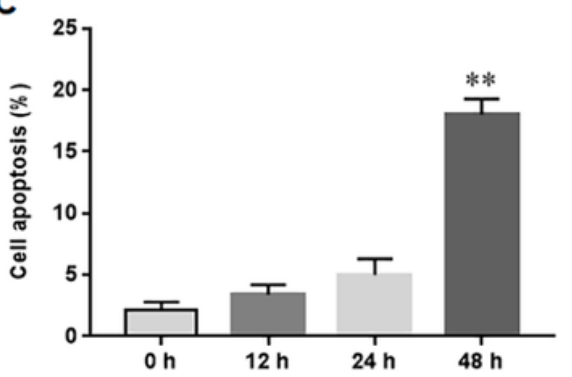

F

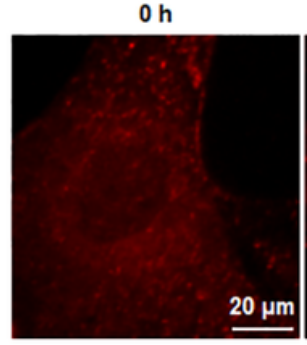

B

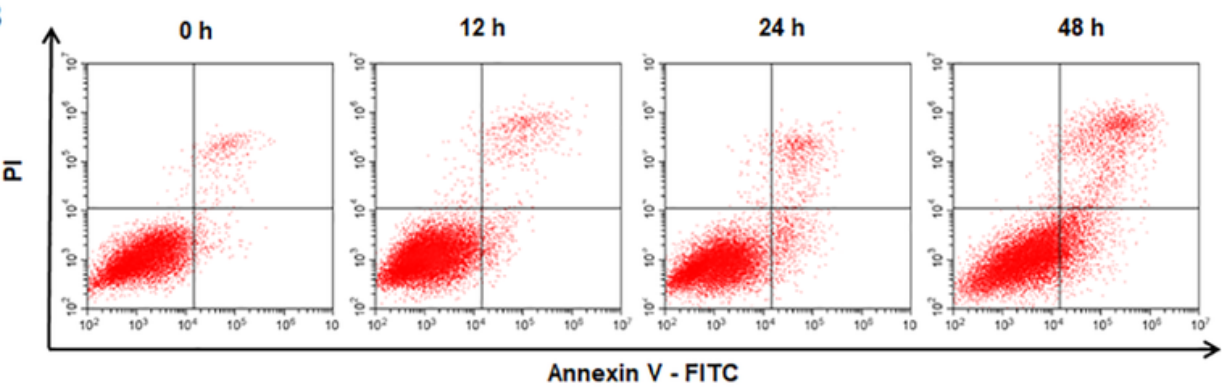

D

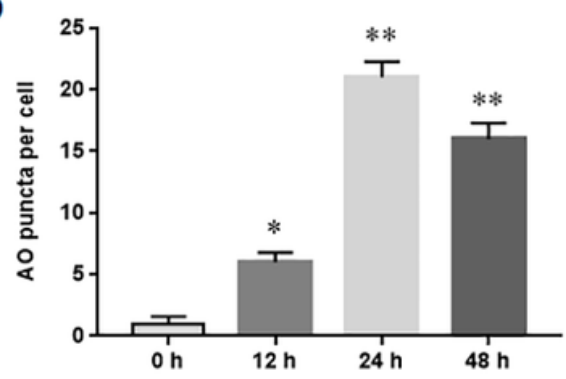

E

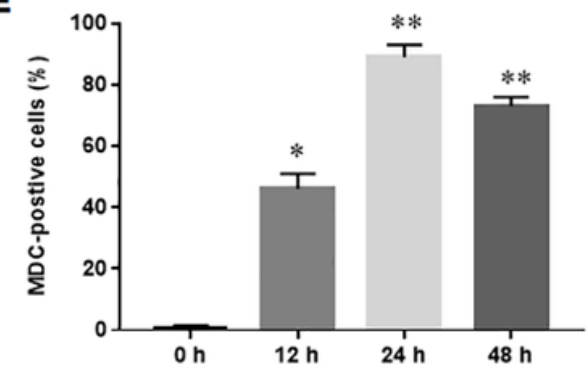

$48 \mathrm{~h}$

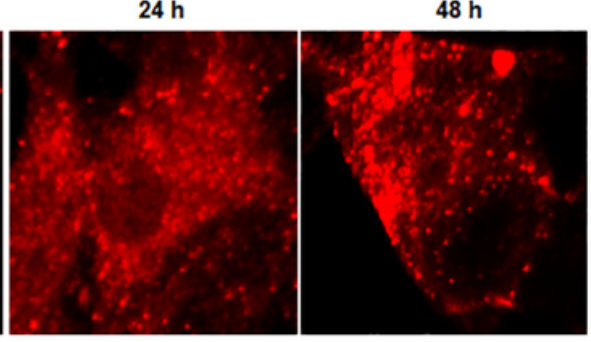

G

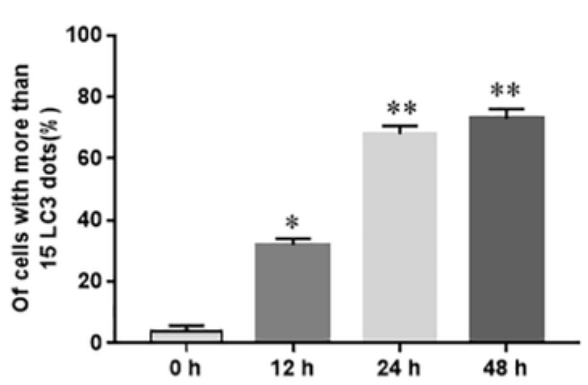

Figure 2

Silencing RHPN1-AS1 induces G2/M cell cycle arrest and switches apoptosis and autophagy in prostate cancer cells. PC3 cells were transfected with sh-RHPN1-AS1 or sh-NC for $48 \mathrm{~h}$, and next cultured at $37^{\circ} \mathrm{C}$, and the cells were collected at 12, 24 and 48 h, respectively. (A) Cell cycle distribution was analyzed by flow cytometry, $N=4$. (B and C) Cell apoptosis was detected with flow cytometry assay, N=4. Autophagy activity was determined with acridine orange (AO) (D) and monodansylcadaverine (MDC) staining (E) or immunofluorescence staining for LC3 puncta ( $F$ and $G), N=4$. The difference of the samples between the two groups were analyzed with independent sample t-test. Compared with $0 \mathrm{~h}, * \mathrm{P}<0.05, * \star \mathrm{P}<0.01$. 
A

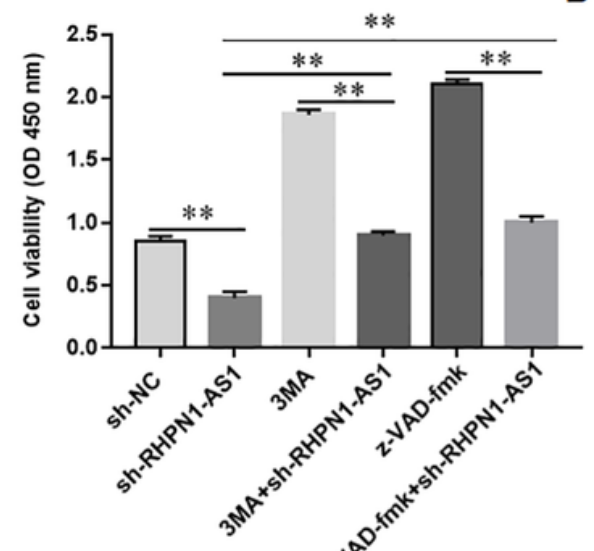

D

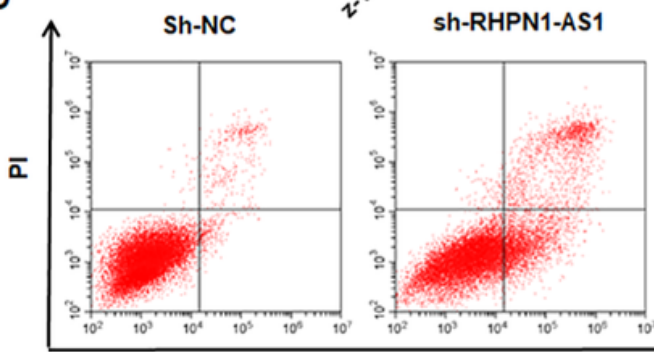

E

$$
\text { LC3-I }
$$

cleaved-caspase-3

cleaved-PARP

$\beta$-actin

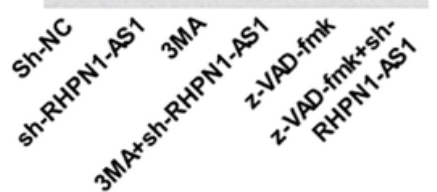

B

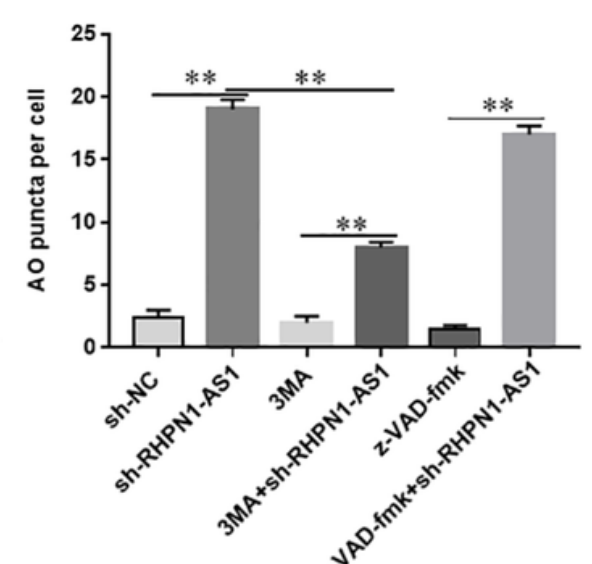

3MA+sh-RHPN1-AS1
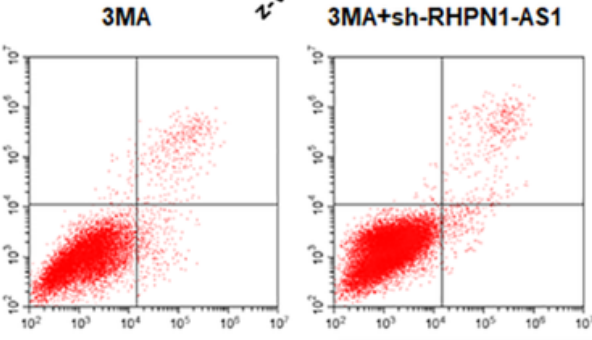

Annexin V - FITC

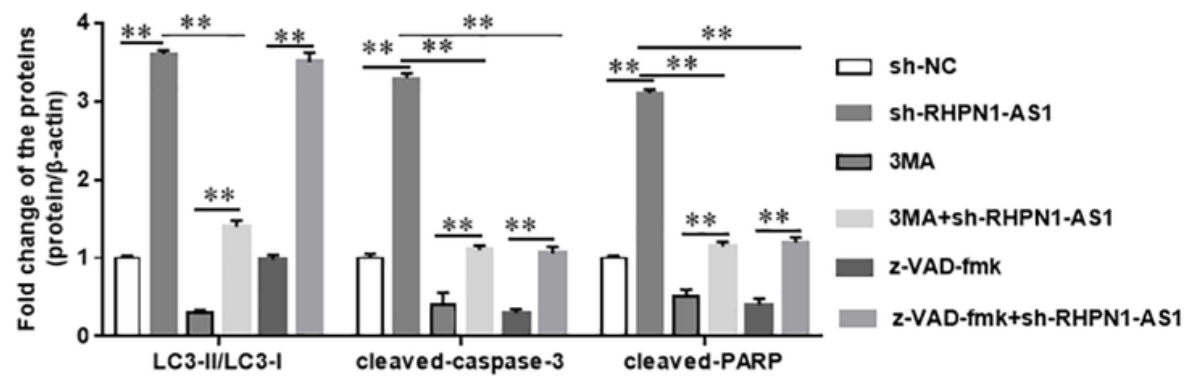

\section{Figure 3}

Silencing RHPN1-AS1 induces autophagy contributes to caspase-dependent apoptosis in prostate cancer cells. PC3 cells were transfected with or without sh-RHPN1-AS1 for $48 \mathrm{~h}$ after $2 \mathrm{~h}$ of pretreatment with 5 mM 3MA (an inhibitor of early autophagy) or $20 \mu \mathrm{m}$ z-VAD-fmk (an irreversible pan-caspase inhibitor). (A) Cell viability was detected by CCK-8 assay, $\mathrm{N}=5$. (B) Autophagy activity was determined with acridine orange ( $A O), N=4$. ( $C$ and $D$ ) Cell apoptosis was detected with flow cytometry assay, $N=4$. (E) The relative protein expression levels of autophagy and apoptosis were measured with Western blotting, $\mathrm{N}=4$. The difference of the samples between the multigroup comparisons of the means were analyzed with the variance test. ${ }^{\star} \mathrm{P}<0.05,{ }^{*} \mathrm{P}<0.01$. 
A

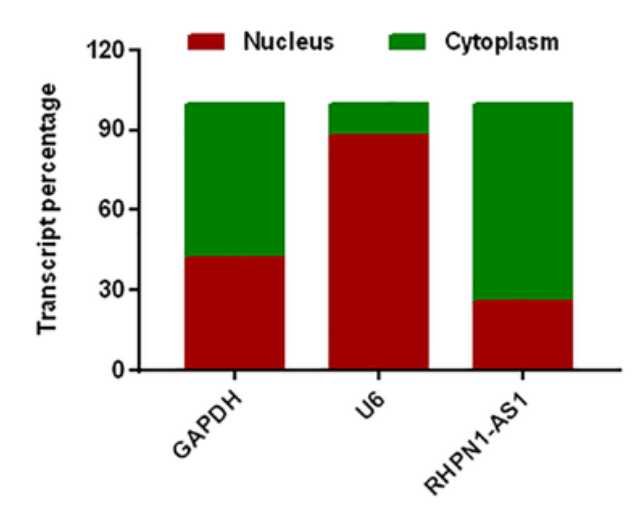

D

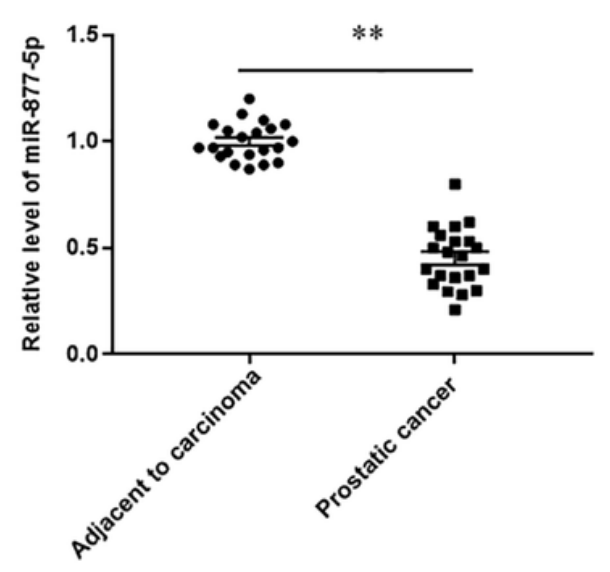

B

RHPN1-AS1 mutant 3' UTR

RHPN1-AS1 wild type 3' UTR

miR-7-5p

C

E
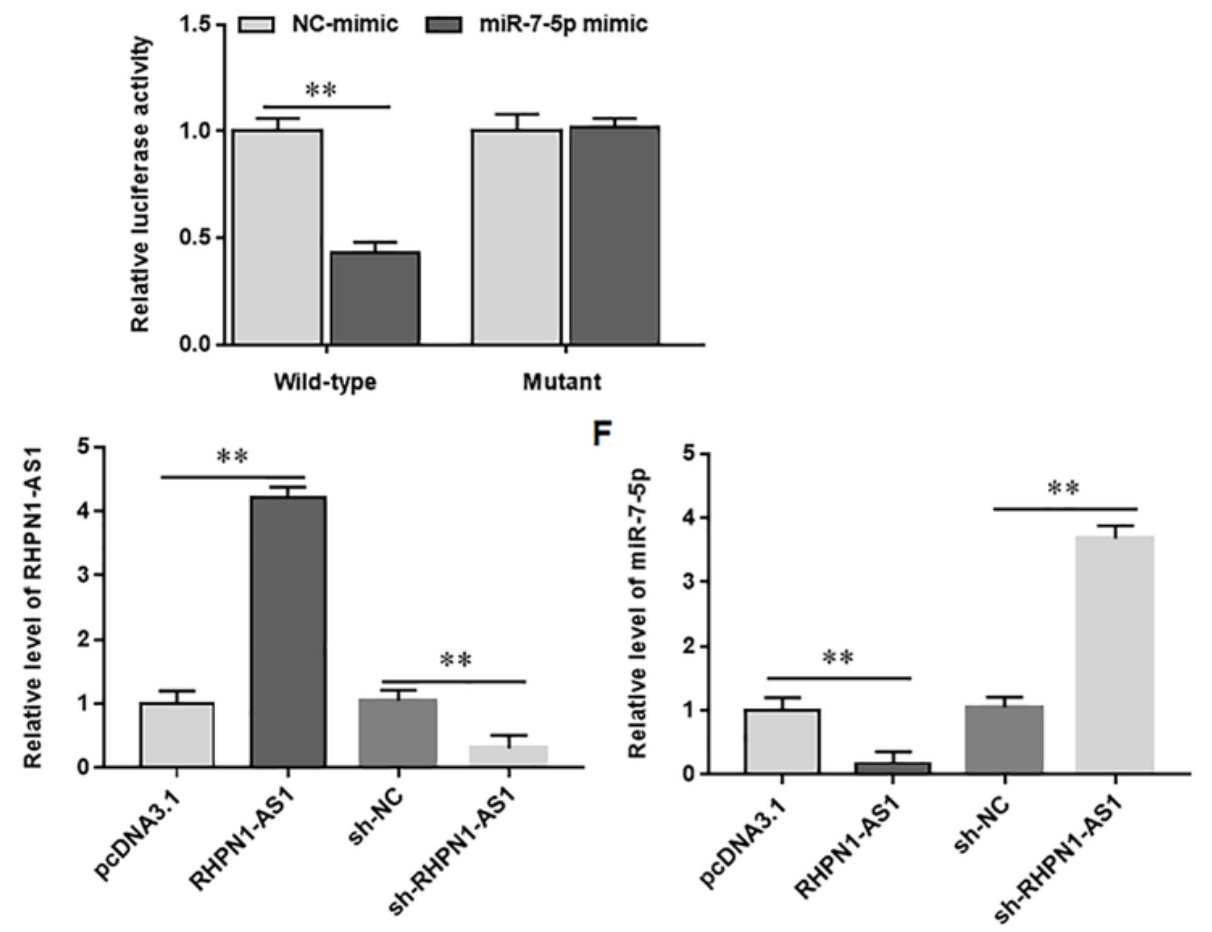

\section{Figure 4}

RHPN1-AS1 binds to miR-7-5p and downregulates miR-7-5p expression. (A) The expression of TRG-AS1 in cytoplasm and nucleus of PC3 cells was detected with RT-qPCR assay. (B) The binding target of RHPN1AS1 and miR-7-5p was predicted by StarBase. (C) The luciferase activity was measured with dual luciferase reporter gene assay. (D) The expression of miR-7-5p was detected with RT-qPCR in prostate cancer tissues and adjacent normal tissues, $\mathrm{N}=21$. PC3 cells were transfected with RHPN1-AS1 overexpression vector (RHPN1-AS1) or sh-RHPN1-AS1 or their control for $48 \mathrm{~h}$, and then the expression of RHPN1-AS1 (E) and miR-7-5p (F) were detected with RT-qPCR, N=5. The difference of the samples between the two groups were analyzed with independent sample t-test, and the analysis of variance test was used to evaluate multigroup comparisons of the means. ${ }^{*} \mathrm{P}<0.05,{ }^{\star} * \mathrm{P}<0.01$. 
A

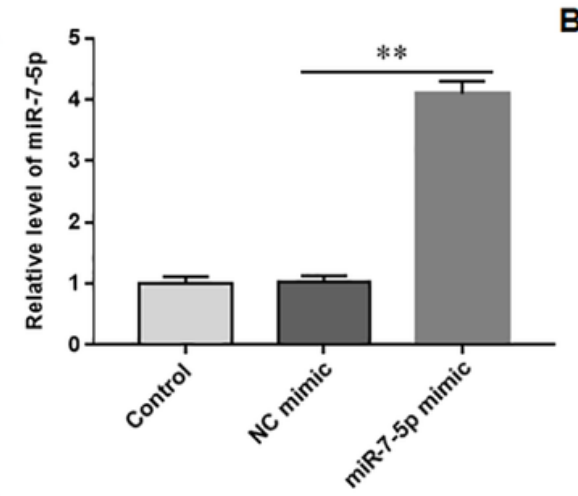

D

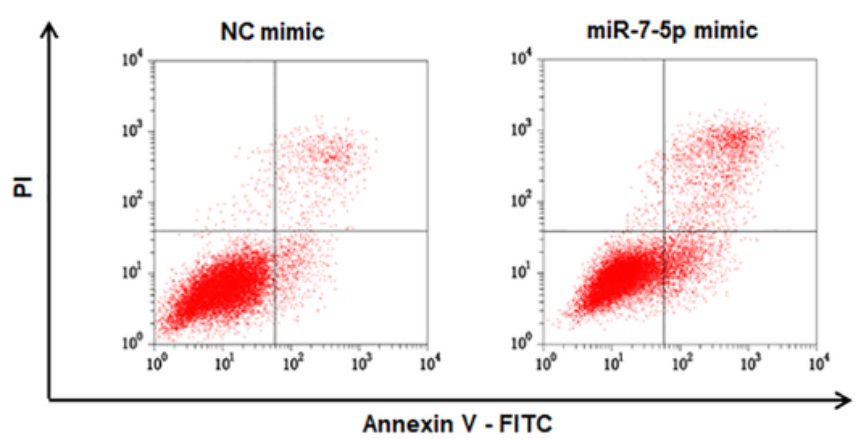

C

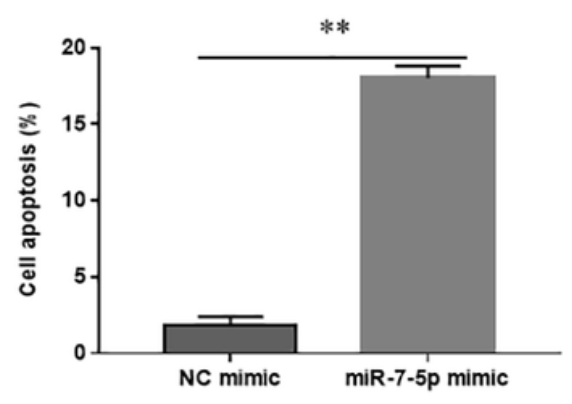

E
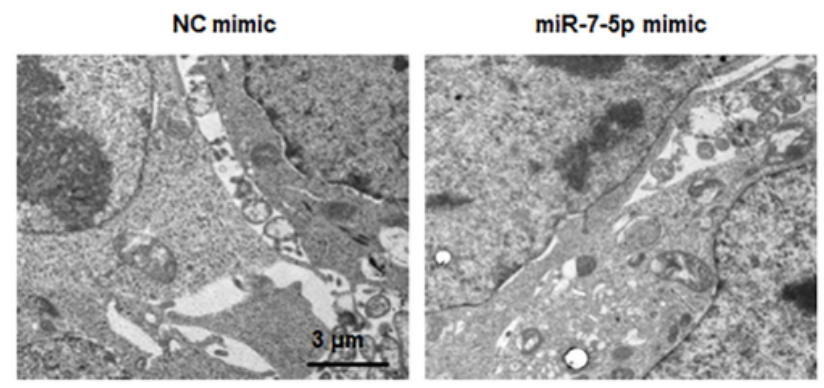

\section{Figure 5}

Overexpression of miR-7-5p inhibits prostate cancer cell proliferation and promotes autophagy and apoptosis. PC3 cells were transfected with miR-7-5p mimic or NC mimic for $48 \mathrm{~h}$, and then the cells were collected. (A) the expression of miR-7-5p was detected with RT-qPCR, N=5. (B) Cell viability was detected by CCK-8 assay, $N=5$. ( $C$ and $D$ ) Cell apoptosis was detected with flow cytometry assay, $N=4$. (E) Transmission electron microscope images show the ultrastructure of PC3 cells (TEM, ×6000). The difference of the samples between the multigroup comparisons of the means were analyzed with the variance test. Compared with $\mathrm{NC}$ mimic, ${ }^{*} \mathrm{P}<0.05$, ${ }^{\star} \mathrm{P}<0.01$. 
A

EGFR mutant 3' UTR

EGFR wild type 3 ' UTR

miR-7-5p
5'... GAGCACAAGCCACAACGCGGCGA...

5'... GAGCACAAGCCACAAGUCUUCCA...

3' ... UUUGUUUUAGUGAUCAGAAGGU...
D

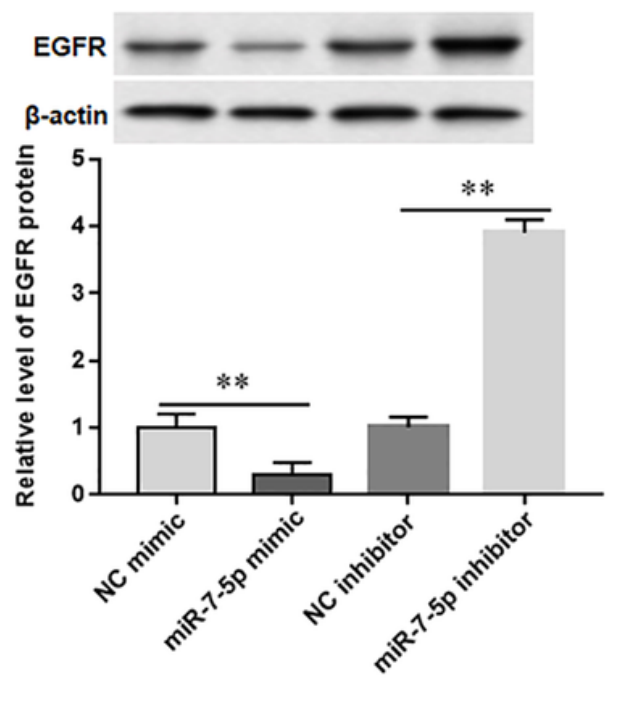

B

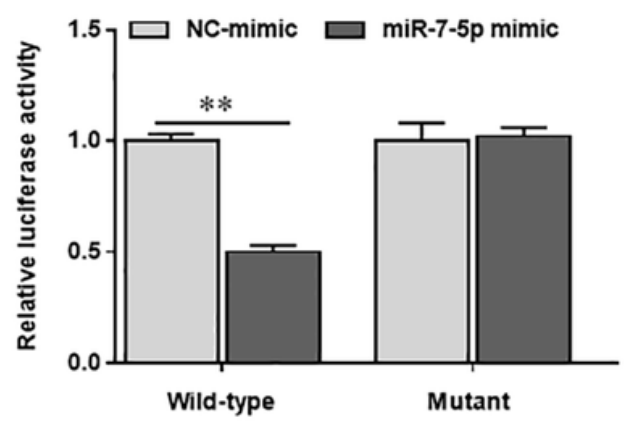

C

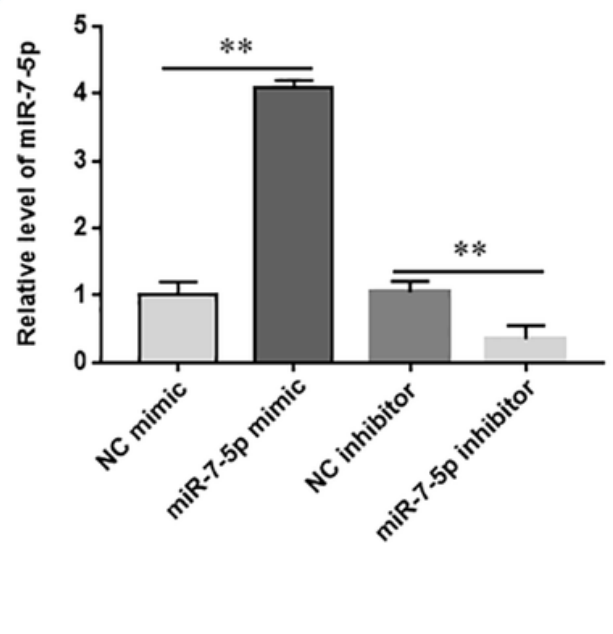

Figure 6

EGFR is a target of miR-7-5p and is regulated by miR-7-5p. (A) The binding target of EGFR and miR-7-5p was predicted by StarBase. (B) The luciferase activity was measured with dual luciferase reporter gene assay. PC3 cells were transfected with miR-7-5p mimic or miR-7-5p inhibitor or their control for $48 \mathrm{~h}$, and then the cells were collected. (C) The expression of miR-7-5p was detected with RT-qPCR, N=5. (D) The protein expression levels of EGFR were measured with Western blotting, $N=4$. (E) PC3 cells were transfected with RHPN1-AS1 overexpression vector (RHPN1-AS1) and/or miR-7-5p mimic or their control for $48 \mathrm{~h}$, and then the protein expression levels of EGFR were measured with Western blotting, $\mathrm{N}=4$. The difference of the samples between the two groups were analyzed with independent sample t-test, and the analysis of variance test was used to evaluate multigroup comparisons of the means. ${ }^{*} P<0.05,{ }^{* *} \mathrm{P}<$ 0.01 . 
A

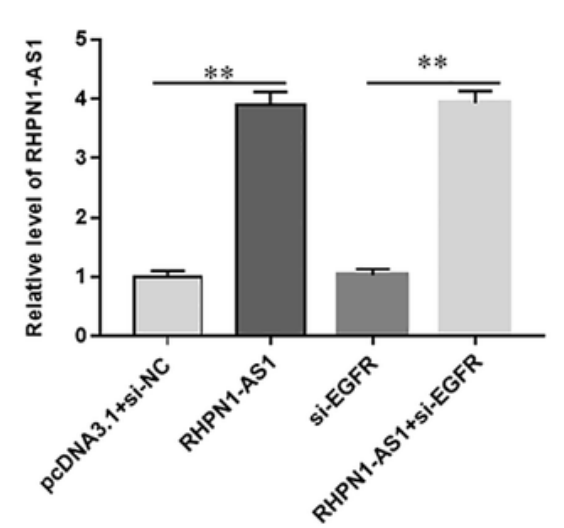

D $\square$ pcDNA3.1+si-NC RHPN1-AS1

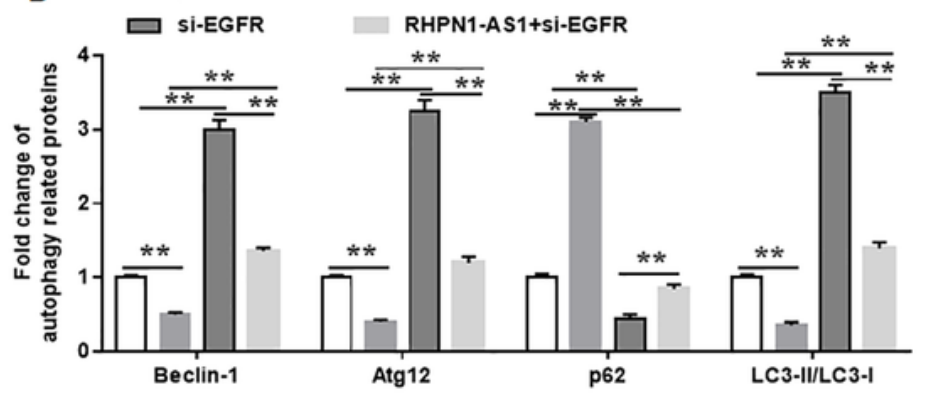

G $\square$ pCDNA3.1+Si-NC $\square$ RHPN1-AS1

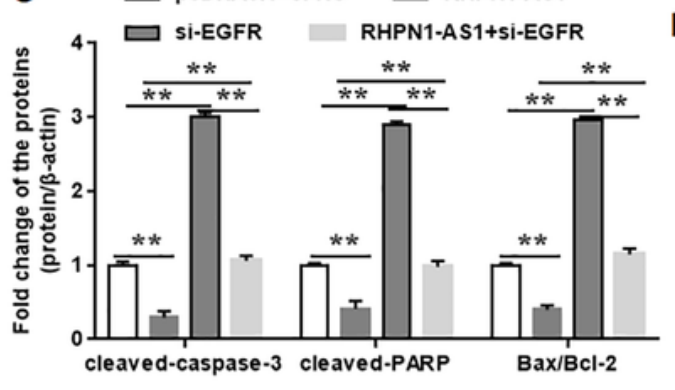

I

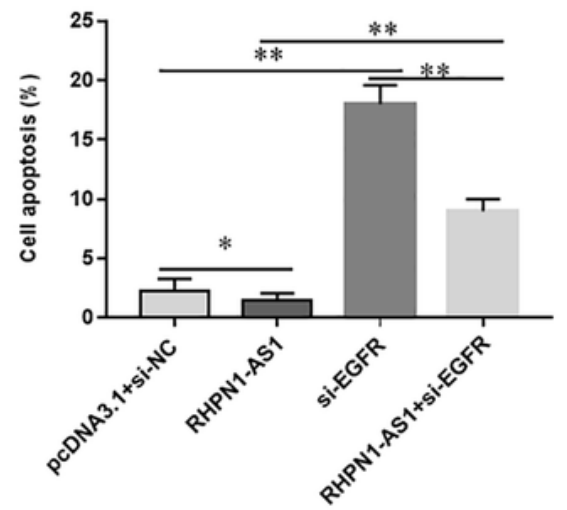

H

\section{J}

B

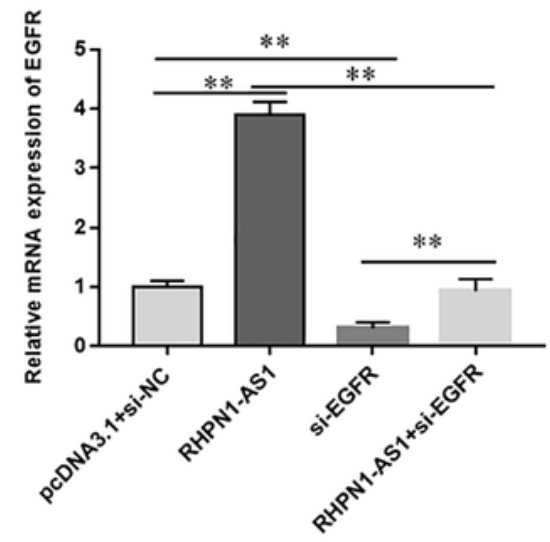

E
C

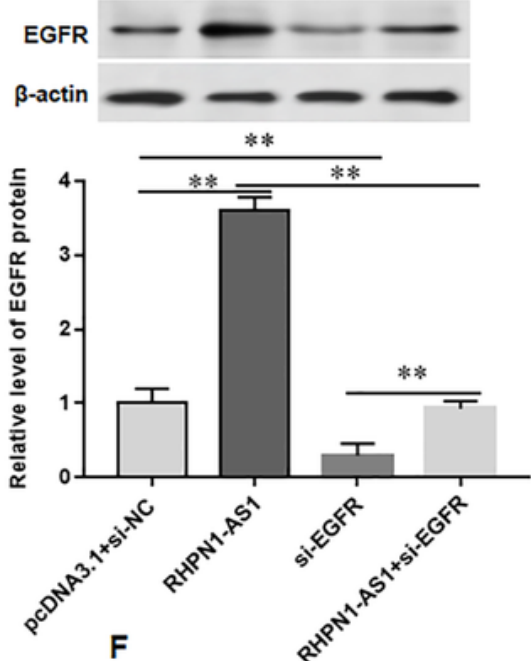

$F$
Beclin-1 - - -

$$
\text { Atg12 }
$$

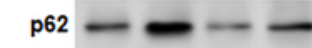

LC3.I $\longrightarrow=-\longrightarrow$

LC3-II

$\beta$-actin

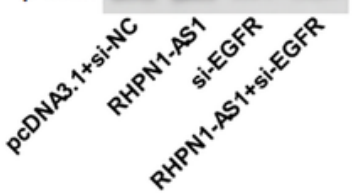

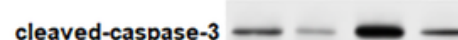

cleaved-PARP - - -

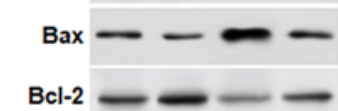

$\beta$-actin --

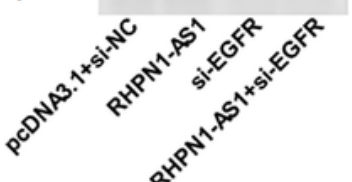

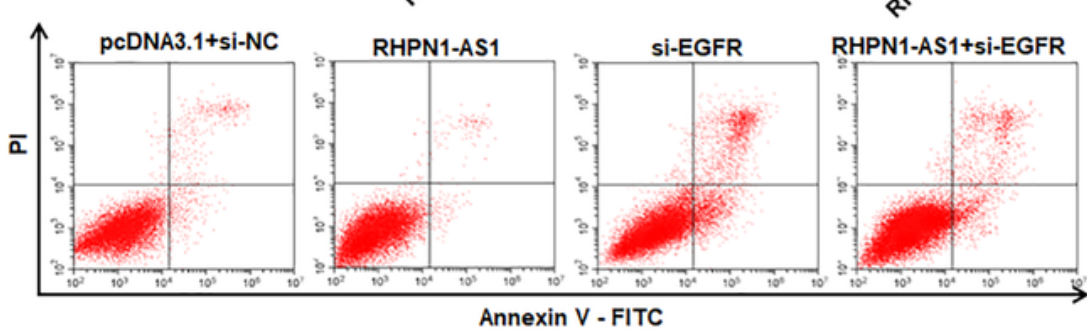
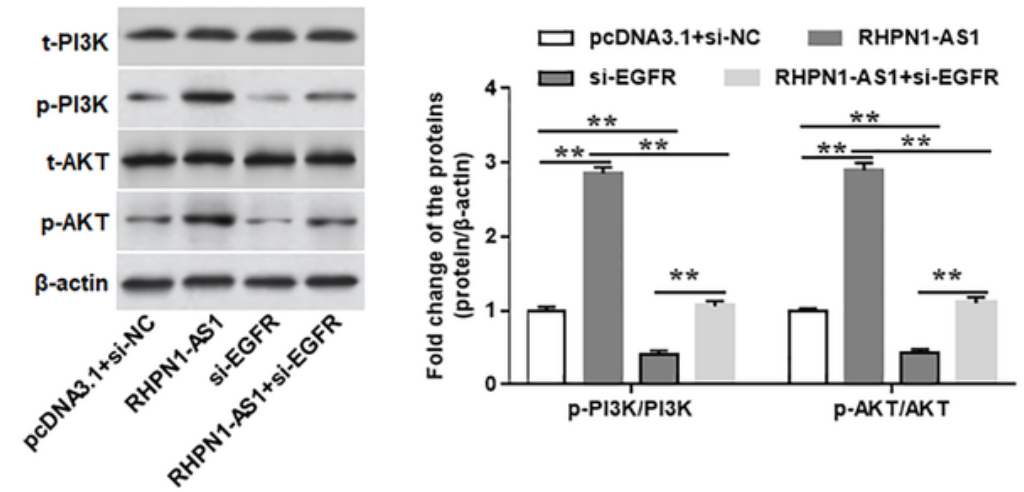

\section{Figure 7}

Silencing EGFR counteracts the inhibitory effect of RHPN1-AS1 on prostate cancer cell apoptosis. PC3 cells were transfected with RHPN1-AS1 overexpression vector (RHPN1-AS1) and/or si-EGFR or their control for $48 \mathrm{~h}$, and then the cells were collected. The expression of RHPN1-AS1 (A) and miR-7-5p (B) was detected with RT-qPCR, N=5. (C) The EGFR protein expression levels were measured with Western blotting, $N=4$. ( $D$ and $E$ ) The relative protein levels of autophagy were measured with Western blotting, 
$\mathrm{N}=4$. ( $\mathrm{F}$ and $\mathrm{G}$ ) The relative protein levels of apoptosis were measured with Western blotting, $\mathrm{N}=4$. ( $\mathrm{H}$ and I) Cell apoptosis was detected with flow cytometry assay, $\mathrm{N}=4$. (J) The protein expression levels of PI3K and AKT were detected with Western blotting, $\mathrm{N}=4$. The difference of the samples between the multigroup comparisons of the means were analyzed with the variance test. ${ }^{*} P<0.05, * * P<0.01$.

$\square$ PCDNA3.1

RHPN1-AS1

RHPN1-AS1+GSK2126458

A

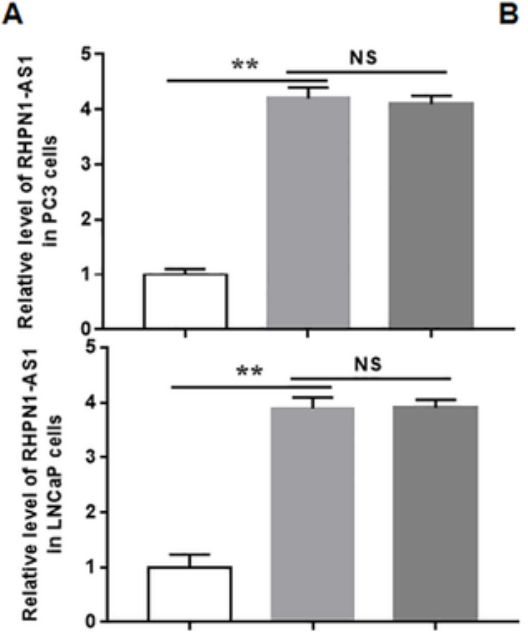

D
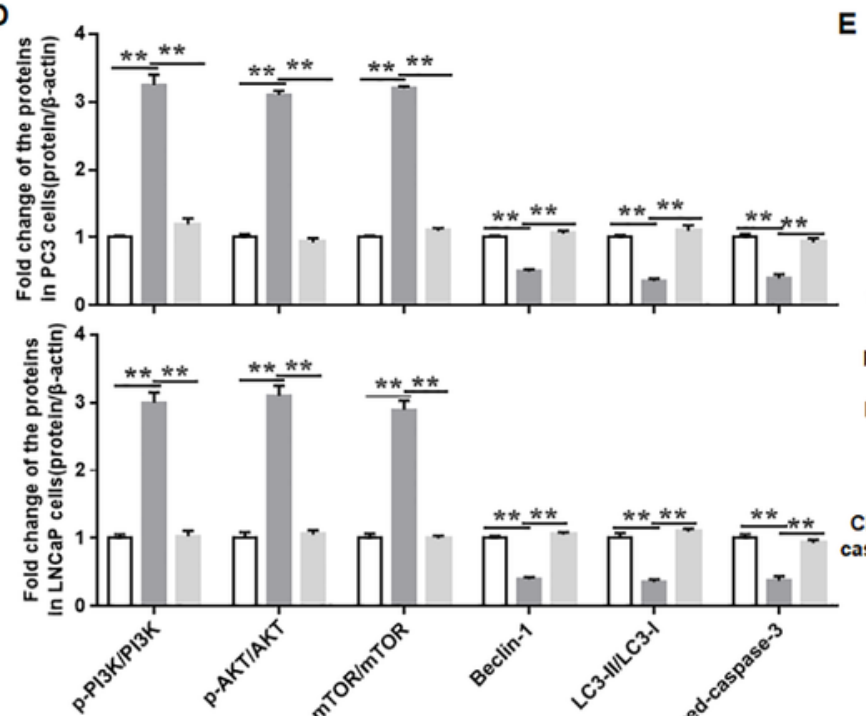

G

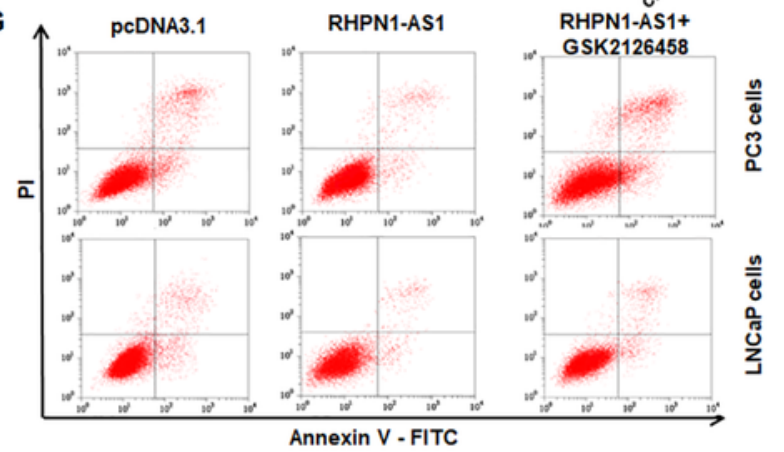

B
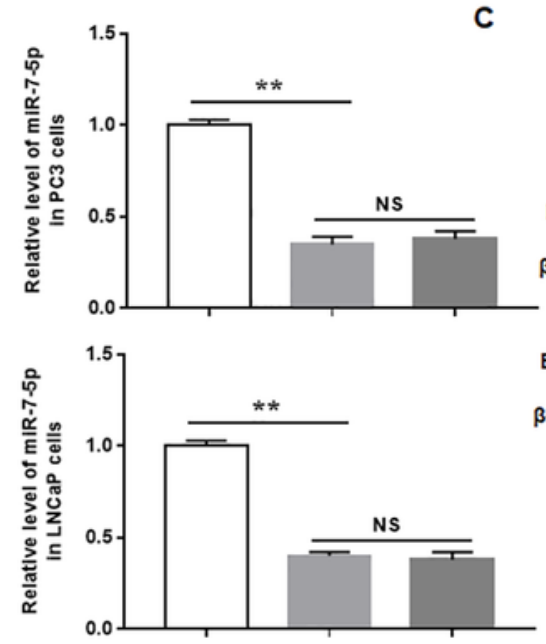

E
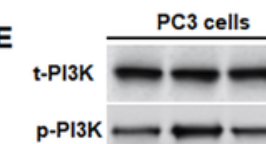

$\mathrm{t}-\mathrm{AKT} \longrightarrow$

$\mathrm{p}-\mathrm{AKT} \longrightarrow$

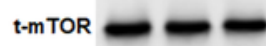

p-mTOR

Beclin-1 $=-\ldots$

LC3-I $=$

LC3-I

Cleaved-

caspase-3
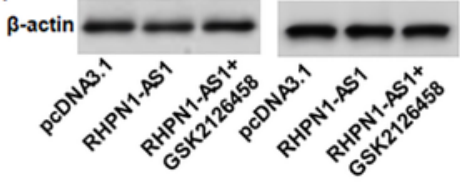

H
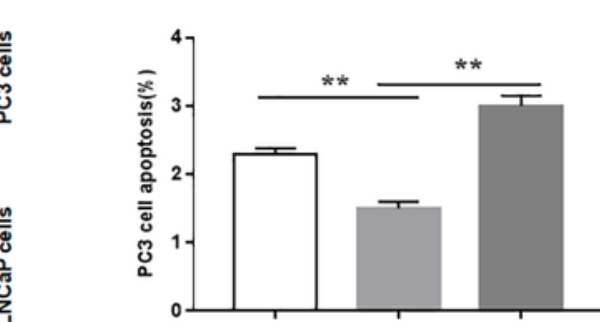

LNCaP cells F
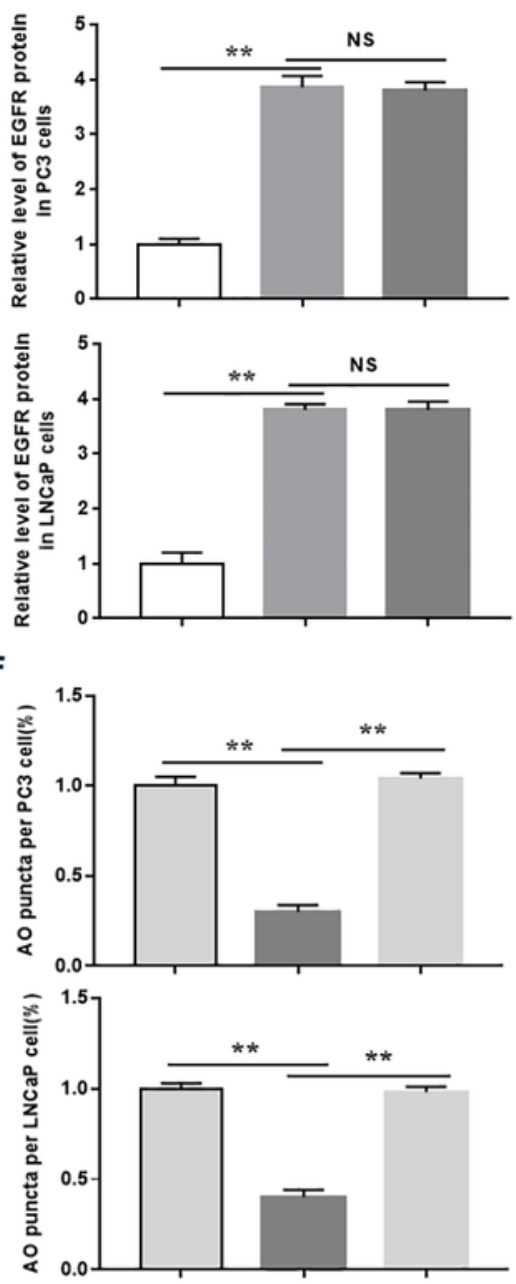

Figure 8 
RHPN1-AS1 inhibits prostate cancer cell apoptosis through the PI3K/AKT/mTOR pathway. PC3 and LNCaP cells were transfected with RHPN1-AS1 overexpression vector (RHPN1-AS1) or pcDNA3.1 empty vector for $48 \mathrm{~h}$ after $2 \mathrm{~h}$ of pretreatment with $3 \mathrm{nM}$ GSK2126458 (an inhibitor of PI3K). The expression of RHPN1-AS1 (A) and miR-7-5p (B) was detected with RT-qPCR, N=5. (C) The EGFR protein expression levels were measured with Western blotting, $N=4$. ( $D$ and $E$ ) The protein expression of the $\mathrm{PI} 3 \mathrm{~K} / \mathrm{AKT} / \mathrm{mTOR}$ pathway key proteins and autophagy relative proteins was detected with Western blotting, $N=4$. ( $F$ ) Autophagy activity was determined with acridine orange ( $A O), N=4$. ( $G$ and $H$ ) Cell apoptosis was detected with flow cytometry assay, $\mathrm{N}=4$. The difference of the samples between the multigroup comparisons of the means were analyzed with the variance test. ${ }^{\star} P<0.05,{ }^{\star *} P<0.01$. 